\title{
Superconvergence Analysis for the Maxwell's Equations in Debye Medium with a Thermal Effect
}

\author{
Changhui Yao ${ }^{1,2, *}$, Dongyang Shi ${ }^{1}$ and Mengmeng $\mathrm{Hou}^{1}$ \\ ${ }^{1}$ School of Mathematics and Statistics, Zhengzhou University, Zhengzhou, Henan 450001, \\ China \\ 2 State Key Laboratory of Space Weather, Chinese Academy of Sciences, Beijing 100190, \\ China
}

Received 29 April 2019; Accepted (in revised version) 18 September 2019

\begin{abstract}
In this paper, a mixed finite element method is investigated for the Maxwell's equations in Debye medium with a thermal effect. In particular, in two dimensional case, the zero order Nédélec element $\left(Q_{01} \times Q_{10}\right)$, the piecewise constant space $Q_{0}$ element, and the bilinear element $Q_{11}$ are used to approximate the electric field $\mathbf{E}$ and the polarization electric field $\mathbf{P}$, the magnetic field $\mathrm{H}$, and the temperature field $u$, respectively. With the help of the high accuracy results, mean-value technique and interpolation postprocessing approach, the convergent rate $\mathcal{O}\left(\tau+h^{2}\right)$ for global superconvergence results are obtained under the time step constraint $\tau=\mathcal{O}\left(h^{1+\gamma}\right), \gamma>0$ by using the linearized backward Euler finite element discrete scheme. At last, a numerical experiment is given to verify the theoretical analysis and the validity of our method.
\end{abstract}

AMS subject classifications: 65N30, 65N15

Key words: Maxwell's equations, thermal effect, error analysis, superconvergence.

\section{Introduction}

In this paper, we consider the following Maxwell's equations:

$$
\begin{array}{ll}
\nabla \times \mathbf{E}=-\frac{\partial \mathbf{B}}{\partial t}, & \nabla \times \mathbf{H}=-\frac{\partial \mathbf{D}}{\partial t}+\mathbf{J}, \\
\nabla \cdot \mathbf{D}=\rho, & \nabla \cdot \mathbf{B}=0,
\end{array}
$$

where $\mathbf{E}$ and $\mathbf{H}$ denote the strengths of the electric and magnetic fields, respectively. $\mathbf{D}$ and $\mathbf{B}$ are the electric and magnetic flux densities, respectively. $\mathbf{J}$ and $\rho$ represent the

*Corresponding author.

Emails: chyao@lsec.cc.ac.cn (C. H. Yao), dy_shi@zzu.edu.cn (D. Y. Shi), 2605822173@qq.com (M. M. Hou) 
current density and the density of free electric charge, respectively. The above equations will be supplemented with the constitutive laws by:

$$
\mathbf{D}=\epsilon_{0} \mathbf{E}+\mathbf{P}, \quad \mathbf{B}=\mu_{0} \mathbf{H}+\mu_{0} \mathbf{M},
$$

where $\mathbf{P}$ and $\mathbf{M}$ represent the electric and magnetic polarization, respectively. $\epsilon_{0}$ and $\mu_{0}$ are the electric permittivity of free space and the magnetic permeability, respectively. We assume $\mathbf{M}=0$ since we can choose to ignore the magnetic effect among the dielectric materials.

Debye medium is one of basic physical concepts when one investigates dielectric in electromagnetic theory and materials science [22]. It is a kind of isotropic dispersive medium, and its permittivity and conductivity are functions of frequency. With the help of the polarization and dielectric relaxation, one can establish phenomenological theory in Debye medium. That is to say, in the process of polarization, microscopic particles complex energy exchange actions can be taken into the following dielectric time parameters. Therefore, numerical studies of Maxwell's equations in Debye medium have attracted considerable attention.

The linear polarization representation originates from the model proposed by Debye [3]. Similar to this representation, in this paper, we consider a linear polarization model

$$
\mathbf{P}_{t}+\frac{1}{t_{0}} \mathbf{P}=\frac{\epsilon_{0}\left(\epsilon_{s}-\epsilon_{\infty}\right)}{t_{0}} \mathbf{E}
$$

where $\epsilon_{s}, \epsilon_{\infty}$ and $t_{0}$ stand by the static relative permittivity, the value of permittivity for an extremely high frequency field and the relaxation time of the dielectric materials, respectively.

Considering the effect of temperature field on electromagnetic field, we use Ohm's law $\mathbf{J}=\sigma(u) \mathbf{E}$ to describe the system, The unknown $u$ is the temperature, the local density of Joule's heat generated by intensive electric waves equals [21]

$$
\mathbf{E} \cdot \mathbf{J}=\mathbf{E} \cdot \sigma(u) \mathbf{E}=\sigma(u)|\mathbf{E}|^{2} .
$$

Thus, from Fourier's law and the conservation of energy, we see that $u$ satisfies

$$
u_{t}-\nabla \cdot(k \nabla u)=\sigma(u)|\mathbf{E}|^{2} r,
$$

where $k$ is the coefficient of thermal conductivity and other physical constants such as density and specific heat have been normalized.

Throughout the paper, we suppose that $\sigma$ is Lipschitz continuous with respect to $u$, which satisfies

$$
0<\sigma_{\min } \leq \sigma \leq \sigma_{\max }
$$


Assumed that $\Omega \subset R^{2}$ is a bounded domain with Lipschitz boundary, we consider the following coupling model

$$
\begin{array}{ll}
\epsilon_{0} \mathbf{E}_{t}+\frac{\epsilon_{0}\left(\epsilon_{s}-\epsilon_{\infty}\right)}{t_{0}} \mathbf{E}+\sigma(u) \mathbf{E}-\nabla \times H-\frac{1}{t_{0}} \mathbf{P}=0, & (X, t) \in \Omega \times(0, T], \\
\mu H_{t}+\nabla \times \mathbf{E}=0, & (X, t) \in \Omega \times(0, T], \\
\mathbf{P}_{t}+\frac{1}{t_{0}} \mathbf{P}=\frac{\epsilon_{0}\left(\epsilon_{s}-\epsilon_{\infty}\right)}{t_{0}} \mathbf{E}, & (X, t) \in \Omega \times(0, T], \\
u_{t}-\nabla \cdot(k \nabla u)=\sigma(u)|\mathbf{E}|^{2}, & (X, t) \in \Omega \times(0, T],
\end{array}
$$

with the initial date

$$
\mathbf{E}(X, 0)=\mathbf{E}_{0}, \quad H(X, 0)=H_{0}, \quad \mathbf{P}(X, 0)=\mathbf{P}_{0}, \quad u(X, 0)=u_{0}, \quad X \in \Omega,
$$

and the perfectly electric boundary condition

$$
\mathbf{E} \times \mathbf{n}=0, \quad u(X, t)=0, \quad(X, t) \in \partial \Omega \times[0, T],
$$

where $\mathbf{n}$ is the outward normal vector on $\partial \Omega$.

As we know, superconvergence analysis has been an interesting field. A sizable amount of researches have been done on the superconvergence of finite element methods for many types of PDEs, such as, the second order elliptic equation [18], parabolic equation [36], Stokes equations [20], nonlinear Sobolev equation [29, 30], Schrödinger equation [5], parabolic equationswith integral two-space-variables condition [6] and so on. There have been a few theoretical results on superconvergence analysis for Maxwell's equation. In [24], the authors studied the superconvergence of the Maxwell's equations in 1994 for the first time. Later, in [27], the authors used the integral identity technique to study this problem once more and improved the result in [24]. The similar result was provied for 2-D and 3-D Maxwell's equations in [7, 8, 11-13, 16, 17]. In [4], the authors discussed the superconvergence of second and third order rectangular edge elements. In [31], the authors studied the superconvergence of nonconforming mixed finite element methods for 3-D time-dependent Maxwell's equations in isotropic cold plasma media. In recent years, Maxwell's equations in Debye medium [26-28,32] have been studied the convergence and superconvergence properties of the nonconforming finite element. Now stochastic collocation methods for Maxwell's equations with random inputs [10] are becoming another popular issue.

The relevant models of Maxwell's equations in the above mentioned studies are linear, and the influence of temperature field on electromagnetic field has been ignored in the process of research. In [35], the coupling system of temperature field and electromagnetic field is studied for the first time, and it is proved that when $\sigma(u)$ is nonnegative and bounded, the weak solution $\mathbf{E}, \mathbf{H}, u$ is existence and uniqueness when $\mathbf{E}(X, t)$, $\mathbf{H}(X, t) \in L^{\infty}\left(0, T ;\left(L^{2}(\Omega)\right)^{3}\right), u(X, t) \in L^{q}\left(0, T ; W_{0}^{1, q}(\Omega)\right), q \in(1,5 / 4)$. However, no relevant 
reports has been found on the finite element method. The similar analysis of existence and uniqueness for nonlinear Maxwell's equations can be found in [33,34].

In this paper, we will focus on the superclose and superconvergence properties of the nonlinear coupled model (1.1a)-(1.3). For convenience, this paper only discusses the case in 2-D. The article is organized as follows. In Section 2, we introduce some notations, the mixed finite element scheme and the variational problem. In Section 3, we give the linearized backward Euler fully discrete scheme and deduce the superclose estimates of order $\mathcal{O}\left(\tau+h^{2}\right)$, where $\tau=\mathcal{O}\left(h^{1+\gamma}\right), \gamma>0$. In Section 4 , the $\mathcal{O}\left(\tau+h^{2}\right)$ order global superconvergence results are obtained with the help of the interpolation postprocessing technique of the coupled model. At last, some numerical results are provided to verify the theoretical analysis, and show the efficiency of the method. Furthermore, it can be verified that when $\gamma>1 / 2$, the conclusion in this paper can be extended to the 3 -D after replacing the mixed Nédélec elements.

\section{Construction of mixed finite elements and variational formulation}

We need the following Sobolev spaces

$$
\begin{aligned}
& H(\text { curl } ; \Omega):=\left\{\mathbf{w}=\left(w_{1}, w_{2}\right) \in\left(L^{2}(\Omega)\right)^{2}: \nabla \times \mathbf{w} \in\left(L^{2}(\Omega)\right)^{2}\right\}, \\
& H_{0}(\text { curl } ; \Omega):=\{\mathbf{w} \in H(\text { curl } ; \Omega): \mathbf{n} \times \mathbf{w}=0 \text { on } \partial \Omega\}, \\
& H^{s}(\operatorname{curl} ; \Omega):=\left\{\mathbf{w} \in\left(H^{s}(\Omega)\right)^{2}: \nabla \times \mathbf{w} \in\left(H^{s}(\Omega)\right)^{2}\right\},
\end{aligned}
$$

where $s>0$, and $\mathbf{n}$ is the unit outer normal to $\Omega$.

The above spaces are equipped with the norms, respectively,

$$
\begin{aligned}
& \|\mathbf{w}\|_{H(\text { curl } ; \Omega)}^{2}:=\|\mathbf{w}\|_{\left(L^{2}(\Omega)\right)^{2}}^{2}+\|\nabla \times \mathbf{w}\|_{\left(L^{2}(\Omega)\right)^{2},}^{2} \\
& \|\mathbf{w}\|_{H^{s}(\operatorname{curl} ; \Omega)}^{2}:=\|\mathbf{w}\|_{H^{s}(\operatorname{curl} ; \Omega)}^{2}+\|\nabla \times \mathbf{w}\|_{H^{s}(\operatorname{curl} ; \Omega)}^{2} .
\end{aligned}
$$

Now, we consider that the weak formulation of system (1.1a)-(1.3) in two-dimention. For any $t>0$, find $(\mathbf{E}(t), H(t), \mathbf{P}(t), u(t)) \in H_{0}($ curl $; \Omega) \times L^{2}(\Omega) \times H($ curl $; \Omega) \times H_{0}^{1}(\Omega)$, such that

$$
\begin{array}{ll}
\epsilon_{0}\left(\mathbf{E}_{t}, \phi\right)+\frac{\epsilon_{0}\left(\epsilon_{s}-\epsilon_{\infty}\right)}{t_{0}}(\mathbf{E}, \phi)+(\sigma(u) \mathbf{E}, \phi)-(H, \nabla \times \phi) & \\
\quad-\frac{1}{t_{0}}(\mathbf{P}, \phi)=0, & \forall \phi \in H_{0}(\text { curl } ; \Omega), \\
\mu\left(H_{t}, \psi\right)+(\nabla \times \mathbf{E}, \psi)=0, & \forall \psi \in L^{2}(\Omega), \\
\left(\mathbf{P}_{t}, \mathbf{w}\right)+\frac{1}{t_{0}}(\mathbf{P}, \mathbf{w})=\frac{\epsilon_{0}\left(\epsilon_{s}-\epsilon_{\infty}\right)}{t_{0}}(\mathbf{E}, \mathbf{w}), & \forall \mathbf{w} \in H(\text { curl; } \Omega), \\
\left(u_{t}, v\right)+(k \nabla u, \nabla v)=\left(\sigma(u)|\mathbf{E}|^{2}, v\right), & \forall v \in H_{0}^{1}(\Omega) . \\
\mathbf{E}(X, 0)=\mathbf{E}_{0}, \quad \mathbf{H}(X, 0)=\mathbf{H}_{0}, \quad \mathbf{P}(X, 0)=\mathbf{P}_{0}, \quad u(X, 0)=u_{0}, & X \in \Omega .
\end{array}
$$


Since $\mathbf{P}$ in (2.1a)-(2.1d) is the ordinary differential equation with respect to $\mathbf{E}$, the existence and uniqueness is similar to [35], and we do not pay much attention to it here.

Assume that $\Omega$ is a rectangle in the $X=(x, y)$ plane with edges parallel to the coordinate axes. Let $\mathcal{T}_{h}$ be a rectangular subdivision of $\Omega$ satisfying the regular condition. Given $K \in \mathcal{T}_{h}$, we denote the lengths of edges parallel to $x$-axis and $y$-axis by $2 h_{x, K}, 2 h_{y, K}$, respectively. Set

$$
h_{K}=\max \left\{h_{x, K}, h_{y, K}\right\} \quad \text { and } \quad h=\max _{K \in \mathcal{T}_{h}}\left\{h_{K}\right\} .
$$

The finite element spaces $\mathbf{N}_{h}, W_{h}, V_{h}$ are defined by

$$
\begin{aligned}
& \mathbf{N}_{h}=\left\{\mathbf{E}=\left(E_{1}, E_{2}\right) \in H(\text { curl } ; \Omega) ;\left.\mathbf{E}\right|_{K} \in Q_{01}(K) \times Q_{10}(K), \forall K \in \mathcal{T}_{h}\right\}, \\
& W_{h}=\left\{w \in L^{2}(\Omega) ;\left.w\right|_{K} \in Q_{0}(K), \forall K \in \mathcal{T}_{h}\right\}, \\
& V_{h}=\left\{v ;\left.v\right|_{K} \in Q_{11}(K), \forall K \in \mathcal{T}_{h}\right\}, \quad V_{0}^{h}=\left\{v ; v \in V_{h},\left.v\right|_{\partial \Omega}=0\right\},
\end{aligned}
$$

where

$$
\begin{aligned}
& H(\operatorname{curl} ; \Omega)=\left\{\mathbf{E} \in\left[L^{2}(\Omega)\right]^{2}, \nabla \times \mathbf{E}=\frac{\partial E_{2}}{\partial x}-\frac{\partial E_{1}}{\partial y}\right\}, \\
& Q_{i j}=\operatorname{span}\left\{x^{r} y^{s}, 0 \leq r \leq i, 0 \leq s \leq j\right\} .
\end{aligned}
$$

For $v \in H^{2}(\Omega), \mathbf{E}=\left(E^{1}, E^{2}\right) \in\left(H^{1}(\Omega)\right)^{2}$, we define the associated interpolation operator $\Pi_{h}, R_{h}, I_{h}$ as

$$
\begin{aligned}
& \Pi_{h}: \mathbf{E} \in\left(H^{1}(\Omega)\right)^{2} \rightarrow \boldsymbol{\Pi}_{h} \mathbf{E} \in \mathbf{N}_{h},\left.\quad \Pi_{h}\right|_{K}=\Pi_{K}, \quad \int_{l_{i}}\left(\mathbf{E}-\boldsymbol{\Pi}_{K} \mathbf{E}\right) \cdot \mathbf{n}_{i} d s=0, \\
& R_{h}: w \in L^{2}(\Omega) \rightarrow R_{h} w \in W_{h},\left.\quad R_{h}\right|_{K}=R_{K}, \quad \int_{K}\left(w-R_{K} w\right) r d s=0, \quad \forall r \in W_{h}, \\
& I_{h}: v \in H^{2}(\Omega) \rightarrow I_{h} v \in V_{h},\left.\quad I_{h}\right|_{K}=I_{K}, \quad I_{K} v\left(a_{i}\right)=v\left(a_{i}\right), \quad i=1,2,3,4,
\end{aligned}
$$

respectively, where $\mathbf{n}_{i}$ is the unit tangent vector of $l_{i}$.

We can cite the following interpolation error estimate in $[1,19,23]$

Lemma 2.1. 1. For any $\mathbf{E} \in H^{\alpha}(\operatorname{curl} ; \Omega), \frac{1}{2}<\alpha \leq 1$, we have

$$
\left\|\mathbf{E}-\Pi_{h} \mathbf{E}\right\|_{0}+\left\|\nabla \times\left(\mathbf{E}-\Pi_{h} \mathbf{E}\right)\right\|_{0} \leq C h^{\alpha}\|\mathbf{E}\|_{H^{\alpha}(\text { curl } ; \Omega)},
$$

2. For $0 \leq m \leq l-1$, if $m$ and $p$ satisfy $m \geq 2, p \leq 1$ or $m=1, p>6 / 5$, for $\forall \mathbf{E} \in\left(W^{m+1, p}(K)\right)^{2}$, we have

$$
\left\|\mathbf{E}-\Pi_{h} \mathbf{E}\right\|_{L^{p}(K)} \leq C h^{m+1}\left\|D^{m+1} \mathbf{E}\right\|_{L^{p}(K)} .
$$

In addition, if $m=0, p \leq 2$, for $\forall \mathbf{E} \in\left(W^{1, p}(K)\right)^{2}, D$ curlE $\in L^{s}(K)$, we have

$$
\left\|\mathbf{E}-\Pi_{h} \mathbf{E}\right\|_{L^{p}(K)} \leq C h\left(\|D \mathbf{E}\|_{L^{p}(K)}+h|K|^{\frac{1}{p}-\frac{1}{s}}\|D \operatorname{curl} \mathbf{E}\|_{L^{s}(K)}\right) .
$$


We also have the following the interpolation error estimate: $v \in H^{m+1}(\Omega)$ for $1 \leq m \leq k$, we have

$$
\left\|v-I_{h} v\right\|_{L^{2}(\Omega)}+h\left\|\nabla\left(v-I_{h} v\right)\right\|_{L^{2}(\Omega)} \leq C h^{m+1}\|v\|_{H^{m+1}(\Omega)},
$$

where $C$ is a positive constant independent of both the mesh size $h$ and the time step $\tau$.

Define $\mathbf{f f}=\left(\alpha_{1}, \alpha_{2}\right) \triangleq \mathbf{E}-\boldsymbol{\Pi}_{h} \mathbf{E}=\left(E_{1}-\boldsymbol{\Pi}_{h} E_{1}, E_{2}-\Pi_{h} E_{2}\right)$, then we recall the following two lemmas which can be found in [14] and plays an important role later.

Lemma 2.2. Assume that $u \in H^{3}(\Omega)$, there hold

$$
\int_{K} \nabla\left(u-I_{h} u\right) \nabla v d x d y=\mathcal{O}\left(h^{2}\right)|u|_{3, K}\|v\|_{1, K^{\prime}} \quad \forall v \in V_{h} .
$$

In addition, if $u \in H^{4}(\Omega)$, we have

$$
\int_{K} \nabla\left(u-I_{h} u\right) \nabla v d x d y=\mathcal{O}\left(h^{2}\right)|u|_{4, K}\|v\|_{0, K}, \quad \forall v \in V_{h}^{0} .
$$

Lemma 2.3. For $\mathbf{E} \in\left(H^{2}(\Omega)\right)^{2}, w \in L^{2}(\Omega)$, we have

$$
\begin{array}{ll}
\left(\mathbf{E}-\boldsymbol{\Pi}_{h} \mathbf{E}, \mathbf{p}\right)=\mathcal{O}\left(h^{2}\right)\|\mathbf{E}\|_{2}\|\mathbf{p}\|_{0}, & \forall \mathbf{p} \in \mathbf{N}_{h}, \\
\left(\nabla \times\left(\mathbf{E}-\boldsymbol{\Pi}_{h} \mathbf{E}\right), r\right)=0, & \forall r \in W_{h}, \\
\left(w-R_{h} w, \nabla \times \mathbf{p}\right)=0, & \forall \mathbf{p} \in \mathbf{N}_{h}, \\
\left(w-R_{h} w, r\right)=0, & \forall r \in W_{h} .
\end{array}
$$

Lemma 2.4. For all $\mathbf{E}=\left(E_{1}, E_{2}\right) \in\left(H^{2}(\Omega)\right)^{2}$, there hold

$$
\left(\alpha_{j}, v\right)=\mathcal{O}\left(h^{2}\right)\left\|E_{j}\right\|_{2}\|v\|_{1}, \quad(j=1,2), \quad \forall v \in V_{h} .
$$

Proof. First of all, we introduce two error functions:

$$
E(x)=\frac{1}{2}\left(\left(x-x_{K}\right)^{2}-h_{x, K}^{2}\right), \quad F(y)=\frac{1}{2}\left(\left(y-y_{K}\right)^{2}-h_{y, K}^{2}\right) .
$$

$\forall v \in V_{h}$, it easy to see that

$$
v(x, y)=v\left(x_{K}, y_{K}\right)+\left(x-x_{K}\right) v_{x}\left(x_{K}, y_{K}\right)+\left(y-y_{K}\right) v_{y}\left(x_{K}, y_{K}\right)+\left(x-x_{K}\right)\left(y-y_{K}\right) v_{x y}
$$

holds. have

By using the properties of $E(x), F(y)$ and the definition of the element $Q_{01} \times Q_{10}$, we

$$
\int_{K} \alpha_{1} d x d y=\int_{K} \alpha_{1} F^{\prime \prime}(y) d x d y=-\int_{K} \alpha_{1 y} F^{\prime}(y) d x d y=\int_{K} E_{1 y y} F(y) d x d y,
$$

which causes

$$
\begin{aligned}
\int_{K} \alpha_{1} v\left(x_{K}, y_{K}\right) d x d y=\int_{K} & E_{1 y y} F(y)\left[v(x, y)-\left(x-x_{K}\right) v_{x}\left(x_{K}, y_{K}\right)\right. \\
& \left.-\left(y-y_{K}\right) v_{y}\left(x_{K}, y_{K}\right)-\left(x-x_{K}\right)\left(y-y_{K}\right) v_{x y}\right] d x d y .
\end{aligned}
$$


With the help of interpolation error estimation and inverse inequality $[9,14]$, we have

$$
\int_{K} \alpha_{1} v\left(x_{K}, y_{K}\right) d x d y=\mathcal{O}\left(h^{2}\right)\left|E_{1}\right|_{2}\|v\|_{1} .
$$

Similarly, we have

$$
\begin{aligned}
& \sum_{K} \int_{K} \alpha_{1}\left(x-x_{K}\right) v_{x}\left(x_{K}, y_{K}\right) d x d y=\mathcal{O}\left(h^{2}\right)\left|E_{1}\right|_{2}\|v\|_{1}, \\
& \sum_{K} \int_{K} \alpha_{1}\left(y-y_{K}\right) v_{y}\left(x_{K}, y_{K}\right) d x d y=\mathcal{O}\left(h^{2}\right)\left|E_{1}\right|_{2}\|v\|_{1}, \\
& \sum_{K} \int_{K} \alpha_{1}\left(x-x_{K}\right)\left(y-y_{K}\right) v_{x y} d x d y=\mathcal{O}\left(h^{2}\right)\left|E_{1}\right|_{2}\|v\|_{1} .
\end{aligned}
$$

By (2.3)-(2.4), we obtain

$$
\left(\alpha_{1}, v\right)=\mathcal{O}\left(h^{2}\right)\left\|E_{1}\right\|_{2}\|v\|_{1}
$$

Similarly,

$$
\left(\alpha_{2}, v\right)=\mathcal{O}\left(h^{2}\right)\left\|E_{2}\right\|_{2}\|v\|_{1}
$$

The proof is completed.

\section{Backward Euler fully discrete scheme}

In this section, we can turn our attention to the discrete scheme. For positive integer $N$, let $0=t_{0}<t_{1}<\cdots<t_{N}=T$ be a partition of the time interval $[0, T]$ with step length $\tau=\frac{T}{N}$ for some positive integer N. For a sequence of function $\left\{\phi^{n}\right\}_{n=0}^{N}$, we denote $\partial_{\tau} \phi^{n}=\frac{\phi^{n}-\phi^{n-1}}{\tau}$. In the same manner of [28], we use the following linearized backward Euler discrete scheme: for $n=1,2, \cdots, N-1$, seek $\left(\mathbf{E}_{h}^{n}, H_{h}^{n}, \mathbf{P}_{h}^{n}, u_{h}^{n}\right): \in \mathbf{N}_{h} \times W_{h} \times \mathbf{N}_{h} \times V_{h}$, such that

$$
\begin{array}{ll}
\epsilon_{0}\left(\partial_{\tau} \mathbf{E}_{h}^{n}, \phi_{h}\right)+\frac{\epsilon_{0}\left(\epsilon_{s}-\epsilon_{\infty}\right)}{t_{0}}\left(\mathbf{E}_{h}^{n}, \phi_{h}\right)+\left(\sigma\left(u_{h}^{n-1}\right) \mathbf{E}_{h}^{n}, \phi_{h}\right) & \\
-\left(H_{h}^{n}, \nabla \times \phi_{h}\right)-\frac{1}{t_{0}}\left(\mathbf{P}_{h}^{n}, \phi_{h}\right)=0, & \forall \phi_{h} \in \mathbf{N}_{h}, \\
\mu\left(\partial_{\tau} H_{h}^{n}, \psi_{h}\right)+\left(\nabla \times \mathbf{E}_{h}^{n}, \psi_{h}\right)=0, & \forall \psi_{h} \in W_{h}, \\
\left(\partial_{\tau} \mathbf{P}_{h}^{n}, \mathbf{w}_{h}\right)+\frac{1}{t_{0}}\left(\mathbf{P}_{h}^{n}, \mathbf{w}_{h}\right)=\frac{\epsilon_{0}\left(\epsilon_{s}-\epsilon_{\infty}\right)}{t_{0}}\left(\mathbf{E}_{h}^{n-1}, \mathbf{w}_{h}\right), & \forall \mathbf{w}_{h} \in \mathbf{N}_{h}, \\
\left(\partial_{\tau} u_{h}^{n}, v_{h}\right)+\left(k \nabla u_{h}^{n}, \nabla v_{h}\right)=\left(\sigma\left(u_{h}^{n-1}\right)\left|\mathbf{E}_{h}^{n}\right|^{2}, v_{h}\right), & \forall v_{h} \in V_{h},
\end{array}
$$

with the initial approximations:

$$
\mathbf{E}_{h}^{0}=\boldsymbol{\Pi}_{h} \mathbf{E}_{0}, \quad H_{h}^{0}=R_{h} H_{0}, \quad \mathbf{P}_{h}^{0}=\boldsymbol{\Pi}_{h} \mathbf{P}_{0}, \quad u_{h}^{0}=\mathbf{I}_{h} u_{0} .
$$

We give the existence and uniqueness of the solutions of Eqs. (3.1a)-(3.1d). 
Theorem 3.1. For any $n=1,2, \cdots, M$, there exists a unique solution $\left(\mathbf{E}_{h}^{n}, H_{h}^{n}, \mathbf{P}_{h}^{n}, u_{h}^{n}\right): \in \mathbf{N}_{h} \times$ $W_{h} \times \mathbf{N}_{h} \times V_{h}$, to solve Eqs. (3.1a)-(3.1d).

Proof. Notice that $\mathbf{E}_{h}^{n}$ and $\mathbf{P}_{h}^{n}$ are chosen from the same finite element space, here $\mathbf{P}_{h}^{n}$ can be computed as follows:

$$
\mathbf{P}_{h}^{n}=\left[\frac{1}{\tau} \mathbf{P}_{h}^{n-1}+\frac{\epsilon_{0}\left(\epsilon_{s}-\epsilon_{\infty}\right)}{t_{0}} \mathbf{E}_{h}^{n-1}\right]\left(\frac{1}{\tau}+\frac{1}{t_{0}}\right)^{-1}
$$

Substituting (3.2) into (3.1a), we can rewrite this equation as follows:

$$
\begin{aligned}
& \left(\frac{\epsilon_{0}}{\tau}+\frac{\epsilon_{0}\left(\epsilon_{s}-\epsilon_{\infty}\right)}{t_{0}}\right)\left(\mathbf{E}_{h}^{n}, \phi_{h}\right)+\left(\sigma\left(u_{h}^{n-1}\right) \mathbf{E}_{h}^{n}, \phi_{h}\right)-\left(H_{h}^{n}, \nabla \times \phi_{h}\right) \\
= & \frac{1}{t_{0}+\tau}\left(\mathbf{P}_{h}^{n-1}, \phi_{h}\right)+\left(\frac{\epsilon_{0}}{\tau}+\frac{\tau \epsilon_{0}\left(\epsilon_{s}-\epsilon_{\infty}\right)}{t_{0}\left(t_{0}+\tau\right)}\right)\left(\mathbf{E}_{h}^{n-1}, \phi_{h}\right), \quad \forall \phi_{h} \in \mathbf{N}_{\mathbf{h}} .
\end{aligned}
$$

On the other hand, we can rewrite (3.1b) as follows

$$
\frac{\mu}{\tau}\left(H_{h}^{n}, \psi_{h}\right)+\left(\nabla \times \mathbf{E}_{h}^{n}, \psi_{h}\right)=\frac{\mu}{\tau}\left(H_{h}^{n-1}, \psi_{h}\right), \quad \forall \psi_{h} \in W_{h} .
$$

Hence, the backward Euler mixed finite element scheme for (3.1a)-(3.1c) can be realized in practice as follows: at each time step, we first solve a system of (3.3)-(3.4) for $\mathbf{E}_{h}^{n}$ and $H_{h}^{n}$, then update $\mathbf{P}_{h}^{n}$ by (3.2).

Finally, notice that the coefficient matrix for the system of (3.3)-(3.4) can be written as

$$
Q \equiv\left(\begin{array}{cc}
A & -B \\
B^{\prime} & D
\end{array}\right)
$$

where the matrices

$$
\begin{aligned}
& A=\left(\frac{\epsilon_{0}}{\tau}+\frac{\epsilon_{0}\left(\epsilon_{s}-\epsilon_{\infty}\right)}{t_{0}}\right)\left(\mathbf{N}_{\mathbf{h}}, \mathbf{N}_{\mathbf{h}}\right)+\left(\sigma^{\frac{1}{2}}\left(u_{h}^{n-1}\right) \mathbf{N}_{\mathbf{h}}, \sigma^{\frac{1}{2}}\left(u_{h}^{n-1}\right) \mathbf{N}_{\mathbf{h}}\right), \\
& B=\left(W_{h}, \nabla \times \mathbf{N}_{\mathbf{h}}\right), \quad D=\frac{\mu}{\tau}\left(W_{h}, W_{h}\right),
\end{aligned}
$$

and $B^{\prime}$ denotes the transpose of matrix $B$. Here, the determinant of $Q$ can be obtained as

$$
\operatorname{det}(Q)=\operatorname{det}(A) \operatorname{det}\left(D+B^{\prime} A^{-1} B\right),
$$

which is guaranteed to be non-zero. Hence the system of (3.3)-(3.4) is guaranteed to have a unique solution $\left(\mathbf{E}_{h}^{n}, H_{h}^{n}\right)$ at each time step.

The equation of (3.1d) can be written as:

$$
\left(U_{h}^{n}, v_{h}\right)+\tau\left(k \nabla U_{h}^{n}, \nabla v_{h}\right)=\tau\left(\sigma\left(U_{h}^{n-1}\right)\left|\mathbf{E}_{h}^{n}\right|^{2}, v_{h}\right)+\left(U_{h}^{n-1}, v_{h}\right), \quad \forall v_{h} \in V_{h},
$$

it is easy to see that (3.7) have a unique solution by Lax-Milgram Lemma. The proof is completed. 
Theorem 3.2. Let $\left(\mathbf{E}^{n}, H^{n}, \mathbf{P}^{n}, u^{n}\right)$ and $\left(\mathbf{E}_{h}^{n}, \mathbf{P}_{h}^{n}, H_{h}^{n}, u_{h}^{n}\right)$ be the solutions of the problem (2.1a)(2.1d) and (3.1a)-(3.1d) at time $t=t_{n}$, respectively. Assume that

$$
\begin{aligned}
& \mathbf{E} \in L^{\infty}\left(0, T ; H^{2}(\text { curl } ; \Omega)\right) \cap\left(W^{1, \infty}(\Omega)\right)^{2}, \quad \mathbf{P} \in L^{\infty}\left(0, T ; H^{2}(\text { curl } ; \Omega)\right), \\
& H \in L^{\infty}\left(0, T ; L^{2} \Omega\right), \quad u \in L^{2}\left(0, T ; H^{4}(\Omega)\right), \\
& \mathbf{E}_{t}, \mathbf{P}_{t} \in L^{\infty}\left(0, T ; H^{2}(\text { curl } ; \Omega)\right), \quad u_{t} \in L^{\infty}\left(0, T ; H^{2}(\Omega)\right), \\
& \mathbf{E}_{t t}, \mathbf{P}_{t t} \in L^{\infty}\left(0, T ;\left(L^{2}(\Omega)\right)^{2}\right), \quad H_{t t} \in L^{\infty}\left(0, T ; L^{2}(\Omega)\right), \quad u_{t t} \in L^{\infty}\left(0, T ; H_{0}^{1}(\Omega)\right) .
\end{aligned}
$$

We have the following superclose estimates

$$
\begin{array}{r}
\max _{1 \leq n \leq N}\left(\left\|\mathbf{E}_{h}^{n}-\boldsymbol{\Pi}_{h} \mathbf{E}^{n}\right\|_{0}+\left\|H_{h}^{n}-\mathbf{R}_{h} H^{n}\right\|_{0}+\| \mathbf{P}_{h}^{n}\right. \\
-\boldsymbol{\Pi}_{h} \mathbf{P}^{n}\left\|_{0}+\right\| \nabla\left(u_{h}^{n}-\mathbf{I}_{h} u^{n}\right) \|_{0} \leq C\left(\tau+h^{2}\right) .
\end{array}
$$

Proof. Let

$$
\begin{aligned}
& \mathbf{E}^{n}-\mathbf{E}_{h}^{n}=\mathbf{E}^{n}-\boldsymbol{\Pi}_{h} \mathbf{E}^{n}+\boldsymbol{\Pi}_{h} \mathbf{E}^{n}-\mathbf{E}_{h}^{n}=: \alpha^{n}+\beta^{n}, \\
& H^{n}-H_{h}^{n}=H^{n}-\mathbf{R}_{h} H^{n}+\mathbf{R}_{h} H^{n}-H_{h}^{n}=: \xi^{n}+\eta^{n}, \\
& \mathbf{P}^{n}-\mathbf{P}_{h}^{n}=\mathbf{P}^{n}-\boldsymbol{\Pi}_{h} \mathbf{P}^{n}+\boldsymbol{\Pi}_{h} \mathbf{P}^{n}-\mathbf{P}_{h}^{n}=: \theta^{n}+\gamma^{n}, \\
& u^{n}-u_{h}^{n}=u^{n}-I_{h} u^{n}+I_{h} u^{n}-u_{h}^{n}=: \lambda^{n}+\zeta^{n} .
\end{aligned}
$$

At every time level $n$, we can get the following error equations

$$
\begin{aligned}
& \epsilon_{0}(\left.\frac{\beta^{n}-\beta^{n-1}}{\tau}, \phi_{h}\right)+\frac{\epsilon_{0}\left(\epsilon_{s}-\epsilon_{\infty}\right)}{t_{0}}\left(\beta^{n}, \phi_{h}\right)+\left(\sigma\left(u^{n-1}\right) \beta^{n}, \phi_{h}\right)-\left(\eta^{n}, \nabla \times \phi_{h}\right)-\frac{1}{t_{0}}\left(\gamma^{n}, \phi_{h}\right) \\
&=- \epsilon_{0}\left(\frac{\alpha^{n}-\alpha^{n-1}}{\tau}, \phi_{h}\right)+\epsilon_{0}\left(R_{1}^{n}, \phi_{h}\right)-\frac{\epsilon_{0}\left(\epsilon_{s}-\epsilon_{\infty}\right)}{t_{0}}\left(\alpha^{n}, \phi_{h}\right) \\
& \quad+\left(\xi^{n}, \nabla \times \phi_{h}\right)+\frac{1}{t_{0}}\left(\theta^{n}, \phi_{h}\right)-\left(\sigma\left(u^{n-1}\right) \alpha^{n}, \phi_{h}\right)-\left(\left(\sigma\left(u^{n-1}\right)-\sigma\left(u_{h}^{n-1}\right)\right) \mathbf{E}_{h}^{n}, \phi_{h}\right) \\
& \quad-\left(\left(\sigma\left(u^{n}\right)-\sigma\left(u^{n-1}\right)\right) \mathbf{E}^{n}, \phi_{h}\right), \\
& \mu\left(\frac{\eta^{n}-\eta^{n-1}}{\tau}, \psi_{h}\right)+\left(\nabla \times \beta^{n}, \psi_{h}\right)=-\mu\left(\frac{\xi^{n}-\xi^{n-1}}{\tau}, \psi_{h}\right)+\mu\left(R_{2}^{n}, \psi_{h}\right)-\left(\nabla \times \alpha^{n}, \psi_{h}\right), \\
&\left(\frac{\gamma^{n}-\gamma^{n-1}}{\tau}, w_{h}\right)+\frac{1}{t_{0}}\left(\gamma^{n}, w_{h}\right) \\
&=-\left(\frac{\theta^{n}-\theta^{n-1}}{\tau}, w_{h}\right)+\left(R_{3}^{n}, w_{h}\right)-\frac{1}{t_{0}}\left(\theta^{k}, w_{h}\right)+\frac{\epsilon_{0}\left(\epsilon_{s}-\epsilon_{\infty}\right)}{t_{0}}\left(\mathbf{E}^{n}-\mathbf{E}^{n-1}, w_{h}\right) \\
& \quad+\frac{\epsilon_{0}\left(\epsilon_{s}-\epsilon_{\infty}\right)}{t_{0}}\left(\alpha^{n-1}, w_{h}\right)+\frac{\epsilon_{0}\left(\epsilon_{s}-\epsilon_{\infty}\right)}{t_{0}}\left(\beta^{n-1}, w_{h}\right), \\
&\left(\frac{\zeta^{n}-\zeta^{n-1}}{\tau}, v_{h}\right)+\left(k \nabla \zeta^{n}, \nabla v_{h}\right) \\
&=-\left(\frac{\lambda^{n}-\lambda^{n-1}}{\tau}, v_{h}\right)-\left(k \nabla \lambda^{n}, \nabla v_{h}\right)+\left(\left(\sigma\left(u^{n-1}\right)-\sigma\left(u_{h}^{n-1}\right)\right)\left|\mathbf{E}_{h}^{n}\right|^{2}, v_{h}\right) \\
& \quad+\left(\sigma\left(u^{n-1}\right)\left(\mathbf{E}^{n}+\mathbf{E}_{h}^{n}\right)\left(\mathbf{E}^{n}-\mathbf{E}_{h}^{n}\right), v_{h}\right)+\left(\left(\sigma\left(u^{n}\right)-\sigma\left(u^{n-1}\right)\right)\left|\mathbf{E}^{n}\right|^{2}, v_{h}\right)+\left(R_{4}^{n}, v_{h}\right),
\end{aligned}
$$


where, $R_{1}^{n}=D_{\tau} \mathbf{E}^{n}-\mathbf{E}_{t}^{n}, R_{2}^{n}=D_{\tau} H^{n}-H_{t}^{n}, R_{3}^{n}=D_{\tau} \mathbf{P}^{n}-\mathbf{P}_{t}^{n}, R_{4}^{n}=D_{\tau} u^{n}-u_{t}^{n}$, and

$$
\left\|R_{1}^{n}\right\|_{0}^{2}=\left\|\tau^{-1} \int_{t_{n-1}}^{t_{n}}\left(t_{n-1}-t\right) \mathbf{E}_{t t}(t) d t\right\|_{0}^{2} \leq C \tau \int_{t_{n-1}}^{t_{n}}\left\|\mathbf{E}_{t t}(t)\right\|_{0}^{2} d t
$$

Similarly,

$$
\begin{aligned}
& \left\|R_{2}^{n}\right\|_{0}^{2} \leq C \tau \int_{t_{n-1}}^{t_{n}}\left\|H_{t t}(t)\right\|_{0}^{2} d t \\
& \left\|R_{3}^{n}\right\|_{0}^{2} \leq C \tau \int_{t_{n-1}}^{t_{n}}\left\|\mathbf{P}_{t t}(t)\right\|_{0}^{2} d t \\
& \left\|R_{4}^{n}\right\|_{0}^{2} \leq C \tau \int_{t_{n-1}}^{t_{n}}\left\|u_{t t}(t)\right\|_{0}^{2} d t .
\end{aligned}
$$

Choosing $\phi_{h}=\beta^{n}$ in Eq. (3.9a) and $\psi_{h}=\eta^{n}$ in Eq. (3.9b), adding the results together, we have

$$
\begin{aligned}
& \epsilon_{0}\left(\frac{\beta^{n}-\beta^{n-1}}{\tau}, \beta^{n}\right)+\frac{\epsilon_{0}\left(\epsilon_{s}-\epsilon_{\infty}\right)}{t_{0}}\left(\beta^{n}, \beta^{n}\right)+\left(\sigma\left(u^{n-1}\right) \beta^{n}, \beta^{n}\right)+\mu\left(\frac{\eta^{n}-\eta^{n-1}}{\tau}, \eta^{n}\right) \\
=- & \epsilon_{0}\left(\frac{\alpha^{n}-\alpha^{n-1}}{\tau}, \beta^{n}\right)+\epsilon_{0}\left(R_{1}^{n}, \beta^{n}\right)-\frac{\epsilon_{0}\left(\epsilon_{s}-\epsilon_{\infty}\right)}{t_{0}}\left(\alpha^{n}, \beta^{n}\right) \\
& +\left(\xi_{n}, \nabla \times \beta^{n}\right)+\frac{1}{t_{0}}\left(\theta^{n}, \beta^{n}\right)+\frac{1}{t_{0}}\left(\gamma^{n}, \beta^{n}\right)-\mu\left(\frac{\xi^{n}-\xi^{n-1}}{\tau}, \eta^{n}\right) \\
& +\mu\left(R_{2}^{n}, \eta_{n}\right)-\left(\nabla \times \alpha^{n}, \eta^{n}\right)-\left(\sigma\left(u^{n-1}\right) \alpha^{n}, \beta^{n}\right)-\left(\left(\sigma\left(u^{n-1}\right)\right.\right. \\
& \left.\left.-\sigma\left(u_{h}^{n-1}\right)\right) \mathbf{E}_{h}^{n}, \beta^{n}\right)-\left(\left(\sigma\left(u^{n}\right)-\sigma\left(u^{n-1}\right)\right) \mathbf{E}^{n}, \beta^{n}\right) \triangleq \sum_{i=1}^{12} A_{i} .
\end{aligned}
$$

Now, we deal with the left term of (3.10),

$$
\begin{aligned}
& \epsilon_{0}\left(\frac{\beta^{n}-\beta^{n-1}}{\tau}, \beta^{n}\right)+\frac{\epsilon_{0}\left(\epsilon_{s}-\epsilon_{\infty}\right)}{t_{0}}\left(\beta^{n}, \beta^{n}\right)+\left(\sigma\left(u^{n-1}\right) \beta^{n}, \beta^{n}\right)+\mu\left(\frac{\eta^{n}-\eta^{n-1}}{\tau}, \eta^{n}\right) \\
\geq & \frac{\epsilon_{0}}{2 \tau}\left(\left\|\beta^{n}\right\|_{0}^{2}-\left\|\beta^{n-1}\right\|_{0}^{2}\right)+\frac{\epsilon_{0}\left(\epsilon_{s}-\epsilon_{\infty}\right)}{t_{0}}\left\|\beta^{n}\right\|_{0}^{2}+\sigma_{\min }\left\|\beta^{n}\right\|_{0}^{2}+\frac{\mu}{2 \tau}\left(\left\|\eta^{n}\right\|_{0}^{2}-\left\|\eta^{n-1}\right\|_{0}^{2}\right) .
\end{aligned}
$$

For the right term of (3.10), using Schwarz inequality, $\epsilon$-Young inequality and Lemma 2.3, we have the following error estimates

$$
\begin{aligned}
A_{1} & \leq\left(C h^{2} \tau^{-1}\left\|\mathbf{E}^{n}\right\|_{2}-C h^{2} \tau^{-1}\left\|\mathbf{E}^{n-1}\right\|_{2}\right)\left\|\beta^{n}\right\|_{0} \\
& \leq \frac{C h^{4}}{\tau} \int_{t_{n-1}}^{t_{n}}\left\|\mathbf{E}_{t}\right\|_{2}^{2} d t+\frac{\epsilon_{0}\left(\epsilon_{s}-\epsilon_{\infty}\right)}{8 t_{0}}\left\|\beta^{n}\right\|_{0}, \\
A_{2} & \leq C\left\|R_{1}^{n}\right\|_{0}^{2}+\frac{\epsilon_{0}\left(\epsilon_{s}-\epsilon_{\infty}\right)}{8 t_{0}}\left\|\beta^{n}\right\|_{0} \leq C \tau \int_{t_{n-1}}^{t_{n}}\left\|\mathbf{E}_{t t}(t)\right\|_{0}^{2} d t+\frac{\epsilon_{0}\left(\epsilon_{s}-\epsilon_{\infty}\right)}{8 t_{0}}\left\|\beta^{n}\right\|_{0}^{2}, \\
A_{3} & \leq \frac{\epsilon_{0}\left(\epsilon_{s}-\epsilon_{\infty}\right)}{t_{0}}\left(C h^{2}\left\|\mathbf{E}^{n}\right\|_{2}\left\|\beta^{n}\right\|_{0}\right) \leq C h^{4}\left\|\mathbf{E}^{n}\right\|_{2}^{2}+\frac{\epsilon_{0}\left(\epsilon_{s}-\epsilon_{\infty}\right)}{8 t_{0}}\left\|\beta^{n}\right\|_{0}^{2}, \\
A_{4} & =A_{7}=A_{9}=0,
\end{aligned}
$$




$$
\begin{aligned}
& A_{5} \leq C h^{2}\left\|\mathbf{P}^{n}\right\|_{2}\left\|\beta^{n}\right\|_{0} \leq C h^{4}\left\|\mathbf{P}^{n}\right\|_{2}^{2}+\frac{\epsilon_{0}\left(\epsilon_{s}-\epsilon_{\infty}\right)}{8 t_{0}}\left\|\beta^{n}\right\|_{0}^{2}, \\
& A_{6} \leq C\left\|\gamma^{n}\right\|_{0}^{2}+\frac{\epsilon_{0}\left(\epsilon_{s}-\epsilon_{\infty}\right)}{8 t_{0}}\left\|\beta^{n}\right\|_{0}^{2}, \\
& A_{8} \leq C\left\|R_{2}^{n}\right\|_{0}^{2}+C\left\|\eta^{n}\right\|_{0}^{2} \leq C \tau \int_{t_{n-1}}^{t_{n}}\left\|H_{t t}(t)\right\|_{0}^{2} d t+C\left\|\eta^{n}\right\|_{0}^{2}, \\
& A_{12} \leq C\left\|u^{n}-u^{n-1}\right\|_{0}\left\|\mathbf{E}^{n}\right\|_{0, \infty}\left\|\beta^{n}\right\|_{0} \leq C \tau \int_{t_{n-1}}^{t_{n}}\left\|u_{t}(t)\right\|_{0}^{2} d t+\frac{\epsilon_{0}\left(\epsilon_{s}-\epsilon_{\infty}\right)}{8 t_{0}}\left\|\beta^{n}\right\|_{0}^{2},
\end{aligned}
$$

where the estimates $A_{3}, A_{4}, A_{5}, A_{7}, A_{9}$ should employ Lemma 2.3.

For any $\omega \in W^{1, \infty}(K)$, define $\bar{\omega}:=\frac{1}{K} \int_{K} \omega d x d y$ (Lemma 3.4 in [2]), then there holds

$$
\|\omega-\bar{\omega}\|_{0, \infty, K} \leq C h_{K}|\omega|_{1, \infty, K}
$$

Using Lemma 2.4, $A_{10}$ can be estimate as

$$
\begin{aligned}
A_{10} & \leq\left|\sum_{K} \int_{K}\left(\sigma\left(u^{n-1}\right)-\overline{\sigma\left(u^{n-1}\right)}\right) \alpha^{n} \beta^{n} d x d y\right|+\left|\sum_{K} \int_{K} \overline{\sigma\left(u^{n-1}\right)} \alpha^{n} \beta^{n} d x d y\right| \\
& \leq \sum_{K}\left\|\sigma\left(u^{n-1}\right)-\overline{\sigma\left(u^{n-1}\right)}\right\|_{0, \infty, K}\left\|\alpha^{n}\right\|_{0, K}\left\|\beta^{n}\right\|_{0, K}+\sum_{K}\left|\int_{K} \overline{\sigma\left(u^{n-1}\right)} \alpha^{n} \beta^{n} d x d y\right| \\
& \leq C h^{2} \sum_{K}\left\|\mathbf{E}^{n}\right\|_{1, K}\left\|\beta^{n}\right\|_{0, K}+C h^{2} \sum_{K}\left\|\mathbf{E}^{n}\right\|_{2, K}\left\|\beta^{n}\right\|_{0, K} \leq C h^{2} \sum_{K}\left\|\mathbf{E}^{n}\right\|_{2, K}\left\|\beta^{n}\right\|_{0, K} \\
& \leq C h^{2}\left\|\mathbf{E}^{n}\right\|_{2}\left\|\beta^{n}\right\|_{0} \leq C h^{4}\left\|\mathbf{E}^{n}\right\|_{2}^{2}+\frac{\epsilon_{0}\left(\epsilon_{s}-\epsilon_{\infty}\right)}{8 t_{0}}\left\|\beta^{n}\right\|_{0}^{2} .
\end{aligned}
$$

To estimate $A_{11}$, similar to the technique used in [28], we give the following mathematical induction hypothesis.

$$
\left\|\mathbf{E}_{h}^{n}\right\|_{0, \infty} \leq \widetilde{C}, \quad \forall n=0,1, \cdots, M
$$

Then, using (3.13), $A_{11}$ can be estimated by

$$
\begin{aligned}
A_{11} & \leq C\left\|u^{n-1}-u_{h}^{n-1}\right\|_{0}\left\|\mathbf{E}_{h}^{n}\right\|_{0, \infty}\left\|\beta^{n}\right\|_{0} \leq C\left(h^{2}\left\|u^{n-1}\right\|_{2}+\left\|\zeta^{n-1}\right\|_{0}\right)\left\|\beta^{n}\right\|_{0} \\
& \leq C h^{4}\left\|u^{n-1}\right\|_{2}^{2}+C\left\|\zeta^{n-1}\right\|_{0}^{2}+\frac{\epsilon_{0}\left(\epsilon_{s}-\epsilon_{\infty}\right)}{8 t_{0}}\left\|\beta^{n}\right\|_{0}^{2} .
\end{aligned}
$$

Taking the above estimates of $A_{i}, i=1,2, \cdots, 12$ into (3.10) and multiplying by $2 \tau$ and summing up from $1 \leq n \leq M$, notice that $\eta^{0}=0, \beta^{0}=0$, we have

$$
\begin{aligned}
& \left\|\beta^{M}\right\|_{0}^{2}+(\mu-C \tau)\left\|\eta^{M}\right\|_{0}^{2} \\
\leq & C\left(h^{4}+\tau^{2}\right)+C \tau \sum_{n=1}^{M-1}\left\|\eta^{n}\right\|_{0}^{2}+C \tau \sum_{n=1}^{M}\left\|\gamma^{n}\right\|_{0}^{2}+C \tau \sum_{n=1}^{M-1}\left\|\zeta^{n}\right\|_{0}^{2} .
\end{aligned}
$$


To get the estimate of $\left\|\gamma^{n}\right\|_{0}^{2}$, setting $w_{h}=\gamma^{n}$ in (3.9c), we have

$$
\begin{aligned}
& \left(\frac{\gamma^{n}-\gamma^{n-1}}{\tau}, \gamma^{n}\right)+\frac{1}{t_{0}}\left(\gamma^{n}, \gamma^{n}\right) \\
=- & \left(\frac{\theta^{n}-\theta^{n-1}}{\tau}, \gamma^{n}\right)+\left(R_{3}^{n}, \gamma^{n}\right)-\frac{1}{t_{0}}\left(\theta^{n}, \gamma^{n}\right)+\frac{\epsilon_{0}\left(\epsilon_{s}-\epsilon_{\infty}\right)}{t_{0}}\left(\mathbf{E}^{n}-\mathbf{E}^{n-1}, \gamma^{n}\right) \\
& +\frac{\epsilon_{0}\left(\epsilon_{s}-\epsilon_{\infty}\right)}{t_{0}}\left(\alpha^{n-1}, \gamma^{n}\right)+\frac{\epsilon_{0}\left(\epsilon_{s}-\epsilon_{\infty}\right)}{t_{0}}\left(\beta^{n-1}, \gamma^{n}\right) \triangleq \sum_{i=1}^{6} B_{i} .
\end{aligned}
$$

The left-hand side of (3.16) becomes

$$
\left(\frac{\gamma^{n}-\gamma^{n-1}}{\tau}, \gamma^{n}\right) \geq \frac{1}{2 \tau}\left(\left\|\gamma^{n}\right\|_{0}^{2}-\left\|\gamma^{n-1}\right\|_{0}^{2}\right), \quad \frac{1}{t_{0}}\left(\gamma^{n}, \gamma^{n}\right)=\frac{1}{t_{0}}\left\|\gamma^{n}\right\|_{0}^{2}
$$

Now, we need to estimate the terms $B_{i}, i=1,2, \cdots, 6$.

By Schwarz inequality, $\epsilon$-Young inequality and Lemma 2.3,

$$
\begin{aligned}
& B_{1} \leq\left(C h^{2} \tau^{-1}\left\|\mathbf{P}^{n}\right\|_{2}-C h^{2} \tau^{-1}\left\|\mathbf{P}^{n-1}\right\|_{2}\right)\left\|\gamma^{n}\right\|_{0} \leq C h^{4} \tau^{-1} \int_{t_{n-1}}^{t_{n}}\left\|\mathbf{P}_{t}\right\|_{2}^{2} d t+\frac{1}{6 t_{0}}\left\|\gamma^{n}\right\|_{0}, \\
& B_{2} \leq C\left\|R_{3}^{n}\right\|_{0}^{2}+\frac{1}{6 t_{0}}\left\|\gamma^{n}\right\|_{0}^{2} \leq C \tau \int_{t_{n-1}}^{t_{n}}\left\|\mathbf{P}_{t t}(t)\right\|_{0}^{2} d t+\frac{1}{6 t_{0}}\left\|\gamma^{n}\right\|_{0}^{2} \\
& B_{3} \leq C h^{2}\left\|\mathbf{P}^{n}\right\|_{2}\left\|\gamma^{n}\right\|_{0} \leq C h^{4}\left\|\mathbf{P}^{n}\right\|_{2}^{2}+\frac{1}{6 t_{0}}\left\|\gamma^{n}\right\|_{0}^{2} \\
& B_{4} \leq C\left\|\mathbf{E}^{n}-\mathbf{E}^{n-1}\right\|\left\|_{0}\right\| \gamma^{n}\left\|_{0} \leq C \tau \int_{t_{n-1}}^{t_{n}}\right\| \mathbf{E}_{t}(t)\left\|_{0}^{2} d t+\frac{1}{6 t_{0}}\right\| \gamma^{n} \|_{0}^{2} \\
& B_{5} \leq C h^{2}\left\|\mathbf{E}^{n-1}\right\|\left\|_{2}\right\| \gamma^{n}\left\|_{0} \leq C h^{4}\right\| \mathbf{E}^{n-1}\left\|_{2}^{2}+\frac{1}{6 t_{0}}\right\| \gamma^{n} \|_{0}^{2} \\
& B_{6} \leq C\left\|\beta^{n-1}\right\|_{0}\left\|\gamma^{n}\right\|_{0} \leq C\left\|\beta^{n-1}\right\|_{0}^{2}+\frac{1}{6 t_{0}}\left\|\gamma^{n}\right\|_{0}^{2}
\end{aligned}
$$

where the estimates $B_{1}, B_{3}, B_{5}$ should employ Lemma 2.3.

Taking the above estimates of $B_{i}, i=1,2, \cdots, 6$ into (3.16), and multiplying by $2 \tau$ and summing up from $1 \leq n \leq M$, notice that $\gamma^{0}=0$, we have

$$
\left\|\gamma^{M}\right\|_{0}^{2} \leq C\left(h^{4}+\tau^{2}\right)+C \tau \sum_{n=1}^{M-1}\left\|\beta^{n}\right\|_{0}^{2} .
$$

To get the estimate of $\left\|\zeta^{n}\right\|_{0}^{2}$, let $v_{h}=\zeta^{n}$ in $(3.9 \mathrm{~d})$

$$
\begin{aligned}
& \left(\frac{\zeta^{n}-\zeta^{n-1}}{\tau}, \zeta^{n}\right)+\left(k \nabla \zeta^{n}, \nabla \zeta^{n}\right) \\
=-( & \left.\frac{\lambda^{n}-\lambda^{n-1}}{\tau}, \zeta^{n}\right)-\left(k \nabla \lambda^{n}, \nabla \zeta^{n}\right) \\
& +\left(\left(\sigma\left(u^{n-1}\right)-\sigma\left(u_{h}^{n-1}\right)\right)\left|\mathbf{E}_{h}^{n}\right|^{2}, \zeta^{n}\right)+\left(\sigma\left(u^{n-1}\right)\left(\mathbf{E}^{n}+\mathbf{E}_{h}^{n}\right)\left(\mathbf{E}^{n}-\mathbf{E}_{h}^{n}\right), \zeta^{n}\right) \\
& +\left(\left(\sigma\left(u^{n}\right)-\sigma\left(u^{n-1}\right)\right)\left|\mathbf{E}^{n}\right|^{2}, \zeta^{n}\right)+\left(R_{4}^{n}, \zeta^{n}\right) \triangleq \sum_{i=1}^{6} D_{i} .
\end{aligned}
$$


The left-hand side of (3.20) becomes

$$
\left(\frac{\zeta^{n}-\zeta^{n-1}}{\tau}, \zeta^{n}\right)+\left(k \nabla \zeta^{n}, \nabla \zeta^{n}\right) \geq \frac{1}{2 \tau}\left(\left\|\zeta^{n}\right\|_{0}^{2}-\left\|\zeta^{n-1}\right\|_{0}^{2}\right)+k\left|\zeta^{n}\right|_{1}^{2} .
$$

Now, we need to estimate the terms $D_{i}, i=1,2, \cdots, 6$. In fact, it is easy to check that

$$
D_{1} \leq C h^{2} \tau^{-1} \int_{t_{n-1}}^{t_{n}}\left\|u_{t}\right\|_{2} d s\left\|\zeta^{n}\right\|_{0} \leq C h^{4} \tau^{-1} \int_{t_{n-1}}^{t_{n}}\left\|u_{t}\right\|_{2}^{2} d s+C\left\|\zeta^{n}\right\|_{0}^{2} .
$$

Then according to (2.2) of Lemma 2.2, there holds

$$
D_{2} \leq C h^{2}\left\|u^{n}\right\|_{4}\left\|\zeta^{n}\right\|_{0} \leq C h^{4}\left\|u^{n}\right\|_{4}^{2}+C\left\|\zeta^{n}\right\|_{0}^{2} .
$$

By (3.13), $D_{3}$ can be estimated as

$$
\begin{aligned}
D_{3} & \leq C\left\|u^{n-1}-u_{h}^{n-1}\right\|_{0}\left\|\mathbf{E}_{h}^{n}\right\|_{0, \infty}^{2}\left\|\zeta^{n}\right\|_{0} \leq C\left(h^{2}\left\|u^{n-1}\right\|_{2}+\left\|\zeta^{n-1}\right\|_{0}\right)\left\|\zeta^{n}\right\|_{0} \\
& \leq C h^{4}\left\|u^{n-1}\right\|_{2}^{2}+C\left\|\zeta^{n-1}\right\|_{0}^{2}+C\left\|\zeta^{n}\right\|_{0}^{2} .
\end{aligned}
$$

As for $D_{4}$, we rewrite it as

$$
D_{4}=\left(\sigma\left(u^{n-1}\right)\left(\mathbf{E}^{n}+\mathbf{E}_{h}^{n}\right) \cdot \alpha^{n}, \zeta^{n}\right)+\left(\sigma\left(u^{n-1}\right)\left(\mathbf{E}^{n}+\mathbf{E}_{h}^{n}\right) \cdot \beta^{n}, \zeta^{n}\right)=G_{1}+G_{2} .
$$

Furthermore, $G_{1}$ can be written as

$$
\begin{aligned}
G_{1} & =2\left(\sigma\left(u^{n-1}\right) \mathbf{E}^{n} \cdot \alpha^{n}, \zeta^{n}\right)+\left(\sigma\left(u^{n-1}\right)\left(\mathbf{E}_{h}^{n}-\mathbf{E}^{n}\right) \cdot \alpha^{n}, \zeta^{n}\right) \\
& =2\left(\sigma\left(u^{n-1}\right) E_{1}^{n} \cdot \alpha_{1}^{n}, \zeta^{n}\right)+2\left(\sigma\left(u^{n-1}\right) E_{2}^{n} \cdot \alpha_{2}^{n}, \zeta^{n}\right)+\left(\sigma\left(u^{n-1}\right)\left(\mathbf{E}_{h}^{n}-\mathbf{E}^{n}\right) \cdot \alpha^{n}, \zeta^{n}\right) \\
& =J_{1}+J_{2}+J_{3} .
\end{aligned}
$$

For $J_{1}$, due to Lemmas 2.1, 2.4 and $H^{1}(\Omega) \hookrightarrow L^{4}(\Omega)$ in two-dimensional space, we have

$$
\begin{aligned}
& J_{1}=2 \sum_{K} \int_{K}\left[\left(\sigma\left(u^{n-1}\right)-\overline{\sigma\left(u^{n-1}\right)}\right) E_{1}^{n} \alpha_{1}^{n} \zeta^{n}+\overline{\sigma\left(u^{n-1}\right)}\left(E_{1}^{n}-\overline{E_{1}^{n}}\right) \alpha_{1}^{n} \zeta^{n}\right. \\
& \left.+\overline{\sigma\left(u^{n-1}\right)} \overline{E_{1}^{n}} \alpha_{1}^{n} \zeta^{n}\right] d x d y \\
& \leq C \sum_{K}\left\|\sigma\left(u^{n-1}\right)-\overline{\sigma\left(u^{n-1}\right)}\right\|_{0, \infty, K}\left\|E_{1}^{n}\right\|_{0, \infty, K}\left\|\alpha_{1}^{n}\right\|_{0, K}\left\|\zeta^{n}\right\|_{0, K} \\
& +C \sum_{K}\left\|E_{1}^{n}-\overline{E_{1}^{n}}\right\|_{0,2}\left\|\alpha_{1}^{n}\right\|_{0,4}\left\|\zeta^{n}\right\|_{0,4}+C \sum_{K} h_{K}^{2}\left\|E_{1}^{n}\right\|_{2, K}\left|\zeta^{n}\right|_{1, K} \\
& \leq C h^{2} \sum_{K}\left\|E_{1}^{n}\right\|_{1, K}\left\|\zeta^{n}\right\|_{0, K}+C h^{2} \sum_{K}\left\|E_{1}^{n}\right\|_{1,2}\left\|E_{1}^{n}\right\|_{1,4}\left|\zeta^{n}\right|_{1, K}+C h^{2} \sum_{K}\left\|E_{1}^{n}\right\|_{2, K}\left|\zeta^{n}\right|_{1, K} \\
& \leq C h^{2}\left\|E_{1}^{n}\right\|_{2, K}\left|\zeta^{n}\right|_{1, K} \leq C h^{4}\left\|E_{1}^{n}\right\|_{2}^{2}+\frac{k}{3}\left|\zeta^{n}\right|_{1}^{2} .
\end{aligned}
$$

Similarly, we have

$$
J_{2} \leq C h^{4}\left\|E_{2}^{n}\right\|_{2}^{2}+\frac{k}{3}\left|\zeta^{n}\right|_{1}^{2}
$$


Furthermore

$$
\begin{aligned}
J_{3} & \leq C\left\|\mathbf{E}_{h}^{n}-\Pi_{h} \mathbf{E}^{n}\right\|_{0,2}\left\|\alpha^{n}\right\|_{0,4}\left\|\zeta^{n}\right\|_{0,4}+C\left\|\boldsymbol{\Pi}_{h} \mathbf{E}^{n}-\mathbf{E}^{n}\right\|_{0,4}\left\|\alpha^{n}\right\|_{0,4}\left\|\zeta^{n}\right\|_{0,2} \\
& \leq C\left\|\beta^{n}\right\|_{0,2}\left\|\alpha^{n}\right\|_{0,4}\left\|\zeta^{n}\right\|_{0,4}+C h^{2}\left\|\mathbf{E}^{n}\right\|_{1,4}^{2}\left\|\zeta^{n}\right\|_{0} \\
& \leq C\left\|\beta^{n}\right\|_{0,2}\left\|\alpha^{n}\right\|_{0,4}\left\|\zeta^{n}\right\|_{1}+C h^{2}\left\|\mathbf{E}^{n}\right\|_{1,4}^{2}\left\|\zeta^{n}\right\|_{0} \\
& \leq C h\left\|\beta^{n}\right\|_{0,2}\left\|\mathbf{E}^{n}\right\|_{1,4}\left\|\zeta^{n}\right\|_{1}+C h^{2}\left\|\mathbf{E}^{n}\right\|_{1,4}^{2}\left\|\zeta^{n}\right\|_{0} \\
& \leq C h^{4}+C\left\|\beta^{n}\right\|_{0}^{2}+\frac{k}{3}\left|\zeta^{n}\right|_{1}^{2} .
\end{aligned}
$$

For $G_{2}$, we have

$$
\begin{gathered}
G_{2} \leq C\left\|\sigma\left(u^{n-1}\right)\right\|_{0, \infty}\left\|\mathbf{E}^{n}+\mathbf{E}_{h}^{n}\right\|_{0, \infty}\left\|\beta^{n}\right\|_{0}\left\|\zeta^{n}\right\|_{0} \leq C\left\|\beta^{n}\right\|_{0}^{2}+C\left\|\zeta^{n}\right\|_{0}^{2}, \\
D_{5}+D_{6} \leq C\left\|u^{n}-u^{n-1}\right\|_{0}\left\|\mathbf{E}^{n}\right\|_{0, \infty}^{2}\left\|\zeta^{n}\right\|_{0}+C\left\|R_{4}^{n}\right\|_{0}\left\|\zeta^{n}\right\|_{0} \\
\leq C \tau \int_{t_{n-1}}^{t_{n}}\left\|u_{t}(t)\right\|_{0}^{2} d t+C \tau \int_{t_{n-1}}^{t_{n}}\left\|u_{t t}(t)\right\|_{0}^{2} d t+C\left\|\zeta^{n}\right\|_{0}^{2} .
\end{gathered}
$$

Then taking (3.21)-(3.28b) into (3.20), we derive

$$
\begin{aligned}
& \frac{1}{2 \tau}\left(\left\|\zeta^{n}\right\|_{0}^{2}-\left\|\zeta^{n-1}\right\|_{0}^{2}\right) \\
\leq & C h^{4} \tau^{-1} \int_{t_{n-1}}^{t_{n}}\left\|u_{t}\right\|_{2}^{2} d s+C\left(h^{4}+\tau^{2}\right)+C\left\|\zeta^{n}\right\|_{0}^{2}+C\left\|\zeta^{n-1}\right\|_{0}^{2}+C\left\|\beta^{n}\right\|_{0}^{2} .
\end{aligned}
$$

Multiplying by $2 \tau$ and summing up from $n=1, \cdots, M,(1 \leq M \leq N)$, we obtain

$$
(1-C \tau)\left\|\zeta^{M}\right\|_{0}^{2} \leq C\left(h^{4}+\tau^{2}\right)+C \tau \sum_{n=1}^{M-1}\left\|\zeta^{n}\right\|_{0}^{2}+C \tau \sum_{n=1}^{M}\left\|\beta^{n}\right\|_{0}^{2} .
$$

By (3.15), (3.19) and (3.29), choosing proper $\epsilon_{0}, \mu, \tau$, so that $\epsilon_{0}-C \tau, \mu-C \tau, 1-C \tau$ are greater than 0 , and applying discrete Grönwall inequality, we get

$$
\left\|\beta^{M}\right\|_{0}+\left\|\eta^{M}\right\|_{0}+\left\|\gamma^{M}\right\|_{0}+\left\|\zeta^{M}\right\|_{0} \leq C\left(\tau+h^{2}\right) .
$$

Now, let's verify (3.13). First, when $n=0$,

$$
\begin{aligned}
\left\|\mathbf{E}_{h}^{0}\right\|_{0, \infty} & =\left\|\boldsymbol{\Pi}_{h} \mathbf{E}(0)\right\|_{0, \infty} \leq\left\|\boldsymbol{\Pi}_{h} \mathbf{E}(0)-\mathbf{E}(0)\right\|_{0, \infty}+\|\mathbf{E}(0)\|_{0, \infty} \\
& \leq C h\|\mathbf{E}(0)\|_{1, \infty}+\|\mathbf{E}(0)\|_{0, \infty} \leq \widetilde{C}
\end{aligned}
$$

Assume that (3.13) holds true for $n=k-1$, then there holds from (3.30) that

$$
\left\|\boldsymbol{\Pi}_{h} \mathbf{E}^{k-1}-\mathbf{E}_{h}^{k-1}\right\|_{0} \leq C\left(h^{2}+\tau\right) .
$$


Then when $n=k$, we need to verify that $\left\|\mathbf{E}_{h}^{k}\right\|_{0, \infty}<\widetilde{C}$. Moreover, we know that $\left\|\mathbf{E}_{h}(t)\right\|_{0, \infty}$ is a continuous function with respect to $t$, so for a given $\epsilon>0$, there exists $\delta>0$, such that when $\left|t_{k}-t_{k-1}\right|=\tau<\delta$, there holds

$$
\left|\left\|\mathbf{E}_{h}^{k}\right\|_{0, \infty}-\left\|\mathbf{E}_{h}^{k-1}\right\|_{0, \infty}\right| \leq \epsilon .
$$

Let $\epsilon=h$,

$$
\begin{aligned}
\left\|\mathbf{E}_{h}^{k}\right\|_{0, \infty} & \leq\left\|\mathbf{E}_{h}^{k-1}\right\|_{0, \infty}+\left|\left\|\mathbf{E}_{h}^{k}\right\|_{0, \infty}-\left\|\mathbf{E}_{h}^{k-1}\right\|_{0, \infty}\right| \\
& \leq\left\|\mathbf{E}_{h}^{k-1}-\mathbf{\Pi}_{h} \mathbf{E}^{k-1}\right\|_{0, \infty}+\left\|\mathbf{\Pi}_{h} \mathbf{E}^{k-1}-\mathbf{E}^{k-1}\right\|_{0, \infty}+\left\|\mathbf{E}^{k-1}\right\|_{0, \infty}+h \\
& \leq C h^{-1}\left\|\mathbf{E}_{h}^{k-1}-\mathbf{\Pi}_{h} \mathbf{E}^{k-1}\right\|_{0}+C h\left\|\mathbf{E}^{k-1}\right\|_{1, \infty}+\left\|\mathbf{E}^{k-1}\right\|_{0, \infty}+h \\
& \leq C\left(h+h^{-1} \tau\right)+\|\mathbf{E}\|_{L^{\infty}\left(W^{0, \infty}(\Omega)\right)} \leq \widetilde{C}
\end{aligned}
$$

where $\tau=\mathcal{O}\left(h^{1+\gamma}\right), \gamma>0$ is needed and $h$ is sufficiently small such that $C h^{\gamma}<1$. Thus we can choose $\widetilde{C}=1+\|\mathbf{E}\|_{L^{\infty}\left(W^{0, \infty(\Omega)}\right)}$. This implies that the mathematical induction (3.13) holds uniformly for any $n$.

Now, we pay attention to the estimate of $\left|\zeta^{n}\right|_{1}^{2}$. Let $v_{h}=\zeta^{n}-\zeta^{n-1}$ in (3.9d)

$$
\begin{aligned}
& \left(\frac{\zeta^{n}-\zeta^{n-1}}{\tau}, \zeta^{n}-\zeta^{n-1}\right)+\left(k \nabla \zeta^{n}, \nabla\left(\zeta^{n}-\zeta^{n-1}\right)\right) \\
=-( & \left.\frac{\lambda^{n}-\lambda^{n-1}}{\tau}, \zeta^{n}-\zeta^{n-1}\right)-\left(k \nabla \lambda^{n}, \nabla\left(\zeta^{n}-\zeta^{n-1}\right)\right)+\left(\left(\sigma\left(u^{n-1}\right)-\sigma\left(u_{h}^{n-1}\right)\right)\left|\mathbf{E}_{h}^{n}\right|^{2}, \zeta^{n}-\zeta^{n-1}\right) \\
& \quad+\left(\sigma\left(u^{n-1}\right)\left(\mathbf{E}^{n}+\mathbf{E}_{h}^{n}\right)\left(\mathbf{E}^{n}-\mathbf{E}_{h}^{n}\right), \zeta^{n}-\zeta^{n-1}\right)+\left(\left(\sigma\left(u^{n}\right)-\sigma\left(u^{n-1}\right)\right)\left|\mathbf{E}^{n}\right|^{2}, \zeta^{n}-\zeta^{n-1}\right) \\
& \quad+\left(R_{4}^{n}, \zeta^{n}-\zeta^{n-1}\right) \triangleq \sum_{i=1}^{6} F_{i} .
\end{aligned}
$$

The left-hand side of (3.32) becomes

$$
\begin{aligned}
& \left(\zeta^{n}-\zeta^{n-1} \tau, \zeta^{n}-\zeta^{n-1}\right)+\left(k \nabla \zeta^{n}, \nabla\left(\zeta^{n}-\zeta^{n-1}\right)\right) \\
\geq & \frac{1}{\tau}\left(\left\|\zeta^{n}-\zeta^{n-1}\right\|_{0}^{2}+\frac{k}{2}\left(\left|\zeta^{n}\right|_{1}^{2}-\left|\zeta^{n-1}\right|_{1}^{2}\right) .\right.
\end{aligned}
$$

Now, we start to estimate each term $F_{i}, i=1,2, \cdots, 6$. First, in the same way as $D_{1}, D_{2}$ and $D_{3}$, we can check that

$$
\begin{aligned}
F_{1} & \leq C h^{2} \tau^{-1} \int_{t_{n-1}}^{t_{n}}\left\|u_{t}\right\|_{2} d s\left\|\zeta^{n}-\zeta^{n-1}\right\|_{0} \leq C h^{4} \int_{t_{n-1}}^{t_{n}}\left\|u_{t}\right\|_{2}^{2} d s+\frac{1}{6 \tau}\left\|\zeta^{n}-\zeta^{n-1}\right\|_{0}^{2}, \\
F_{2} & \leq C h^{2}\left\|u^{n}\right\|_{4}\left\|\zeta^{n}-\zeta^{n-1}\right\|_{0} \leq C \tau h^{4}+\frac{1}{6 \tau}\left\|\zeta^{n}-\zeta^{n-1}\right\|_{0}^{2} \\
F_{3} & \leq C\left\|u^{n-1}-u_{h}^{n-1}\right\|_{0}\left\|\mathbf{E}_{h}^{n}\right\|_{0, \infty}^{2}\left\|\zeta^{n}-\zeta^{n-1}\right\|_{0} \leq C\left(h^{2}\left\|u^{n-1}\right\|_{0}+\left\|\zeta^{n-1}\right\|_{0}\right)\left\|\zeta^{n}-\zeta^{n-1}\right\|_{0} \\
& \leq C\left(h^{2}+\tau\right)\left\|\zeta^{n}-\zeta^{n-1}\right\|_{0} \leq C \tau\left(h^{4}+\tau^{2}\right)+\frac{1}{6 \tau}\left\|\zeta^{n}-\zeta^{n-1}\right\|_{0}^{2}
\end{aligned}
$$


Second, we rewrite $F_{4}$ as

$$
\begin{aligned}
F_{4}= & \left(\sigma\left(u^{n-1}\right)\left(\mathbf{E}^{n}+\mathbf{E}_{h}^{n}\right)\left(\mathbf{E}^{n}-\mathbf{E}_{h}^{n}\right), \zeta^{n}-\zeta^{n-1}\right) \\
= & \left(\sigma\left(u^{n-1}\right)\left(\mathbf{E}^{n}+\mathbf{E}_{h}^{n}\right) \cdot \alpha^{n}, \zeta^{n}-\zeta^{n-1}\right)+\left(\sigma\left(u^{n-1}\right)\left(\mathbf{E}^{n}+\mathbf{E}_{h}^{n}\right) \cdot \beta^{n}, \zeta^{n}-\zeta^{n-1}\right) \\
= & \left.2\left(\sigma\left(u^{n-1}\right) \mathbf{E}^{n} \cdot \alpha^{n}\right), \zeta^{n}-\zeta^{n-1}\right)+\left(\sigma\left(u^{n-1}\right)\left(\mathbf{E}_{h}^{n}-\mathbf{E}^{n}\right) \cdot \alpha^{n}, \zeta^{n}-\zeta^{n-1}\right) \\
& \quad \quad \quad\left(\sigma\left(u^{n-1}\right)\left(\mathbf{E}^{n}+\mathbf{E}_{h}^{n}\right) \cdot \beta^{n}, \zeta^{n}-\zeta^{n-1}\right) \\
= & \left.\left.2\left(\sigma\left(u^{n-1}\right) E_{1}^{n} \cdot \alpha_{1}^{n}\right), \zeta^{n}-\zeta^{n-1}\right)+2\left(\sigma\left(u^{n-1}\right) E_{2}^{n} \cdot \alpha_{2}^{n}\right), \zeta^{n}-\zeta^{n-1}\right) \\
& \quad+\left(\sigma\left(u^{n-1}\right)\left(\mathbf{E}_{h}^{n}-\mathbf{E}^{n}\right) \cdot \alpha^{n}, \zeta^{n}-\zeta^{n-1}\right)+\left(\sigma\left(u^{n-1}\right)\left(\mathbf{E}^{n}+\mathbf{E}_{h}^{n}\right) \cdot \beta^{n}, \zeta^{n}-\zeta^{n-1}\right) \\
= & F_{41}+F_{42}+F_{43}+F_{44} .
\end{aligned}
$$

Now, we start to estimate each term $F_{4 i},(i=1,2,3,4)$. Due to Lemma 2.1 and Lemma 2.3-2.4, inverse inequality, we have

$$
\begin{aligned}
F_{43} & \leq C\left\|\mathbf{E}_{h}^{n}-\Pi_{h} \mathbf{E}^{n}\right\|_{0,4}\left\|\alpha^{n}\right\|_{0,4}\left\|\zeta^{n}-\zeta^{n-1}\right\|_{0, K}+C\left\|\mathbf{\Pi}_{h} \mathbf{E}^{n}-\mathbf{E}^{n}\right\|_{0,4}\left\|\alpha^{n}\right\|_{0,4}\left\|\zeta^{n}\right\|_{0, K} \\
& \leq C h^{-\frac{1}{2}}\left(h^{2}+\tau\right) C h\left\|\mathbf{E}^{n}\right\|_{1,4}\left\|\zeta^{n}-\zeta^{n-1}\right\|_{0, K}+C h^{2}\left\|\mathbf{E}^{n}\right\|_{1,4}^{2}\left\|\zeta^{n}-\zeta^{n-1}\right\|_{0} \\
& \leq C\left(h^{\frac{5}{2}}+h^{\frac{1}{2}} \tau\right)\left\|\zeta^{n}-\zeta^{n-1}\right\|_{0, K}+C h^{2}\left\|\zeta^{n}-\zeta^{n-1}\right\|_{0} \\
& \leq C \tau\left(h^{4}+\tau^{2}\right)+\frac{1}{6 \tau}\left\|\zeta^{n}-\zeta^{n-1}\right\|_{0}^{2} \\
F_{44} & \leq C\left\|\sigma\left(u^{n-1}\right)\right\|_{0, \infty}\left\|\mathbf{E}^{n}+\mathbf{E}_{h}^{n}\right\|_{0, \infty}\left\|\beta^{n}\right\|_{0}\left\|\zeta^{n}-\zeta^{n-1}\right\|_{0} \leq C\left(h^{2}+\tau\right)\left\|\zeta^{n}-\zeta^{n-1}\right\|_{0} \\
& \leq C \tau\left(h^{4}+\tau^{2}\right)+\frac{1}{6 \tau}\left\|\zeta^{n}-\zeta^{n-1}\right\|_{0}^{2} .
\end{aligned}
$$

For $F_{41}$, using the identity $\left(a^{n}, b^{n}-b^{n-1}\right)=\left(a^{n}, b^{n}\right)-\left(a^{n-1}, b^{n-1}\right)-\left(a^{n}-a^{n-1}, b^{n-1}\right)$, we have

$$
\begin{aligned}
F_{41}=2\left[\left(\sigma\left(u^{n-1}\right) E_{1}^{n} \cdot \alpha_{1}^{n}, \zeta^{n}\right)-\left(\sigma\left(u^{n-2}\right) E_{1}^{n-1} \cdot \alpha_{1}^{n-1}, \zeta^{n-1}\right)\right] \\
\quad-2\left(\sigma\left(u^{n-1}\right) E_{1}^{n} \cdot \alpha_{1}^{n}-\sigma\left(u^{n-2}\right) E_{1}^{n-1} \cdot \alpha_{1}^{n-1}, \zeta^{n-1}\right) \\
=2\left[\left(\sigma\left(u^{n-1}\right) E_{1}^{n} \cdot \alpha_{1}^{n}, \zeta^{n}\right)-\left(\sigma\left(u^{n-2}\right) E_{1}^{n-1} \cdot \alpha_{1}^{n-1}, \zeta^{n-1}\right)\right] \\
\quad-2\left[\left(\left(\sigma\left(u^{n-1}\right)-\sigma\left(u^{n-2}\right)\right) E_{1}^{n} \cdot \alpha_{1}^{n}, \zeta^{n-1}\right)+\left(\sigma\left(u^{n-2}\right)\left(E_{1}^{n}-E_{1}^{n-1}\right) \alpha_{1}^{n}, \zeta^{n-1}\right)\right. \\
\left.\quad+\left(\sigma\left(u^{n-2}\right) E_{1}^{n-1}\left(\alpha_{1}^{n}-\alpha_{1}^{n-1}\right), \zeta^{n-1}\right)\right] .
\end{aligned}
$$

For the third term of the above equation, applying the mean-value inequality, we have

$$
\begin{aligned}
& \quad-2\left(\left(\sigma\left(u^{n-1}\right)-\sigma\left(u^{n-2}\right)\right) E_{1}^{n} \cdot \alpha_{1}^{n} \zeta^{n-1}\right) \\
& =-2 \sum_{K} \int_{K}\left[\left(\sigma^{\prime}(\lambda)-\overline{\sigma^{\prime}(\lambda)}\right)\left(u^{k-1}-u^{k-2}\right) E_{1}^{n} \alpha_{1}^{n} \zeta^{n-1}\right. \\
& \quad+\overline{\sigma^{\prime}(\lambda)}\left(u^{k-1}-u^{k-2}-\overline{u^{k-1}-u^{k-2}}\right) E_{1}^{n} \alpha_{1}^{n} \zeta^{n-1} \\
& \left.\quad+\overline{\sigma^{\prime}(\lambda)} \overline{u^{k-1}-u^{k-2}}\left(E_{1}^{n}-\overline{E_{1}^{n}}\right) \alpha_{1}^{n} \zeta^{n-1}+\overline{\sigma^{\prime}(\lambda)} \overline{u^{k-1}-u^{k-2}} \overline{E_{1}^{n}} \alpha_{1}^{n} \zeta^{n-1}\right] d x d y \\
& \leq \sum_{K} C_{K} h_{K}^{2} \tau\left\|E_{1}^{n}\right\|_{1, K}\left\|\zeta^{n-1}\right\|_{0, K}+\sum_{K} C_{K} h_{K}^{2} \tau\left\|E_{1}^{n}\right\|_{1, K}\left\|\zeta^{n-1}\right\|_{0, K} \\
& \quad+\sum_{K} C_{K} h_{K}^{2} \tau\left\|E_{1}^{n}\right\|_{1, K}\left\|E_{1}^{n}\right\|_{1,4}\left\|\zeta^{n-1}\right\|_{0,4}+\sum_{K} C_{K} h_{K}^{2} \tau\left\|E_{1}^{n}\right\|_{2, K}\left\|\zeta^{n-1}\right\|_{1, K}
\end{aligned}
$$




$$
\leq C h^{2} \tau\left|\zeta^{n-1}\right|_{1} \leq C h^{4} \tau+C \tau\left|\zeta^{n}\right|_{1}^{2},
$$

where $\lambda$ is between at $u^{n-1}$ and $u^{n-2}$.

Similarly, we can derive

$$
\begin{aligned}
& -2\left(\sigma\left(u^{n-2}\right)\left(E_{1}^{n}-E_{1}^{n-1}\right) \alpha_{1}^{n}, \zeta^{n-1}\right) \leq C h^{4} \tau+C \tau\left|\zeta^{n}\right|_{1}^{2}, \\
& -2\left(\sigma\left(u^{n-2}\right) E_{1}^{n-1}\left(\alpha_{1}^{n}-\alpha_{1}^{n-1}\right), \zeta^{n-1}\right) \leq C h^{4} \tau+C \tau\left|\zeta^{n}\right|_{1}^{2} .
\end{aligned}
$$

Therefore, $F_{41}$ reduce to

$$
F_{41} \leq 2\left[\left(\sigma\left(u^{n-1}\right) E_{1}^{n} \cdot \alpha_{1}^{n}, \zeta^{n}\right)-\left(\sigma\left(u^{n-2}\right) E_{1}^{n-1} \cdot \alpha_{1}^{n-1}, \zeta^{n-1}\right)\right]+C h^{4} \tau+C \tau\left|\zeta^{n}\right|_{1}^{2} .
$$

In the same way, we have

$$
F_{42} \leq 2\left[\left(\sigma\left(u^{n-1}\right) E_{2}^{n} \cdot \alpha_{2}^{n}, \zeta^{n}\right)-\left(\sigma\left(u^{n-2}\right) E_{2}^{n-1} \cdot \alpha_{2}^{n-1}, \zeta^{n-1}\right)\right]+C h^{4} \tau+C \tau\left|\zeta^{n}\right|_{1}^{2} .
$$

Furthermore, it follows that

$$
\begin{aligned}
F_{4} \leq 2[( & \left.\left.\sigma\left(u^{n-1}\right) E_{1}^{n} \cdot \alpha_{1}^{n}, \zeta^{n}\right)-\left(\sigma\left(u^{n-2}\right) E_{1}^{n-1} \cdot \alpha_{1}^{n-1}, \zeta^{n-1}\right)\right] \\
& +2\left[\left(\sigma\left(u^{n-1}\right) E_{2}^{n} \cdot \alpha_{2}^{n}, \zeta^{n}\right)-\left(\sigma\left(u^{n-2}\right) E_{2}^{n-1} \cdot \alpha_{2}^{n-1}, \zeta^{n-1}\right)\right] \\
& +C\left(h^{4}+\tau^{2}\right) \tau+C \tau\left|\zeta^{n}\right|_{1}^{2}+\frac{1}{3 \tau}\left\|\zeta^{n}-\zeta^{n-1}\right\|_{0}^{2} .
\end{aligned}
$$

Furthermore, we have

$$
\begin{aligned}
F_{5}+F_{6} & \leq C\left\|u^{n}-u^{n-1}\right\|_{0}\left\|\mathbf{E}^{n}\right\|_{0, \infty}^{2}\left\|\zeta^{n}-\zeta^{n-1}\right\|_{0}+C\left\|R_{4}^{n}\right\|_{0}^{2}\left\|\zeta^{n}-\zeta^{n-1}\right\|_{0} \\
& \leq C \tau^{2} \int_{t_{n-1}}^{t_{n}}\left\|u_{t}(t)\right\|_{0}^{2} d t+C \tau^{2} \int_{t_{n-1}}^{t_{n}}\left\|u_{t t}(t)\right\|_{0}^{2} d t+\frac{1}{6 \tau}\left\|\zeta^{n}-\zeta^{n-1}\right\|_{0}^{2} .
\end{aligned}
$$

Then taking (3.33)-(3.38) into (3.32), and summing up the above inequality and noticing that $\zeta^{0}=0$, we obtain

$$
\begin{aligned}
& \left(\frac{k}{2}-C \tau\right)\left|\zeta^{M}\right|_{1}^{2} \\
\leq & 2\left(\sigma\left(u^{M-1}\right) E_{1}^{M} \cdot \alpha_{1}^{M}, \zeta^{M}\right)+2\left(\sigma\left(u^{M-1}\right) E_{2}^{M} \cdot \alpha_{2}^{M}, \zeta^{M}\right)+C\left(h^{4}+\tau^{2}\right)+C \tau \sum_{n=1}^{M-1}\left|\zeta^{n}\right|_{1}^{2} .
\end{aligned}
$$

By choosing proper $\tau$ so that $\frac{k}{2}-C \tau>0$, applying the similar process as $J_{1}$ for the first and second terms in the right-hand side of the above inequality, we have

$$
\left|\zeta^{M}\right|_{1}^{2} \leq C\left(h^{4}+\tau^{2}\right)+C \tau \sum_{n=1}^{M-1}\left|\zeta^{n}\right|_{1}^{2},
$$

by applying discrete Grönwall inequality, we can complete the proof. 


\section{Global superconvergence analysis}

To obtain global superconvergence, we merge the adjacent four elements $K_{1}, K_{2}, K_{3}, K_{4}$ into one big element $\widetilde{K}=\bigcup_{i=1}^{4} K_{i}$ (Fig. 1) without overlapped.

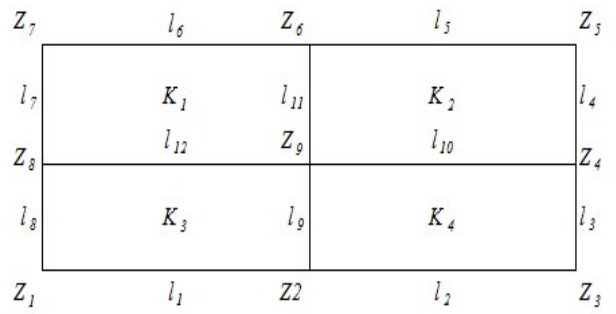

Figure 1: Large unit $\widetilde{K}$.

By use of the postprocessing interpolation operators $\Pi_{2 h^{\prime}}^{2} \mathbf{J}_{2 h^{\prime}}^{2} \mathbf{I}_{2 h}^{2}$ constructed in [14]:

$$
\begin{aligned}
& \left.\Pi_{2 h}^{2} \mathbf{w}\right|_{\widetilde{K}} \rightarrow Q_{11}(\widetilde{K}) \times Q_{11}(\widetilde{K}), \quad \forall \mathbf{w}=\left(w_{1}, w_{2}\right) \in\left(H^{2}(\widetilde{K})\right)^{2}, \quad \widetilde{K} \in \Gamma_{2 h}, \\
& \int_{l_{i}}\left(\mathbf{\Pi}_{2 h}^{2} w_{1}-w_{1}\right) d s=0, \quad i=1,2,5,6 ; \quad \int_{l_{i}}\left(\Pi_{2 h}^{2} w_{2}-w_{2}\right) d s=0, \quad i=3,4,7,8 ; \\
& \left.\mathbf{J}_{2 h}^{2} q\right|_{\widetilde{K}} \in Q_{11}, \quad \int_{e_{i}}\left(\mathbf{J}_{2 h}^{2} q-q\right) d x d y=0, \quad i=1,2,3,4 ; \\
& \left.\mathbf{I}_{2 h}^{2} u\right|_{\widetilde{K}} \in Q_{22}(\widetilde{K}), \quad \forall u \in C(\widetilde{K}), \quad \mathbf{I}_{2 h}^{2} u\left(Z_{i}\right)=u\left(Z_{i}\right), \quad i=1, \cdots, 9,
\end{aligned}
$$

where $C(\widetilde{K})$ is a continuous function space on $\widetilde{K}$, the postprocessing interpolation operators $\Pi_{2 h}^{2}, \mathbf{J}_{2 h}^{2}, \mathbf{I}_{2 h}^{2}$ (details in [14]) satisfy

$$
\begin{aligned}
& \boldsymbol{\Pi}_{2 h}^{2} \boldsymbol{\Pi}_{h} \mathbf{w}=\boldsymbol{\Pi}_{2 h}^{2} \mathbf{w}, \quad \mathbf{J}_{2 h}^{2} \mathbf{J}_{h} q=\mathbf{J}_{2 h}^{2} q, \quad \mathbf{I}_{2 h}^{2} \mathbf{I}_{h} u=\mathbf{I}_{2 h}^{2} u, \\
& \left\|\boldsymbol{\Pi}_{2 h}^{2} \mathbf{w}-\mathbf{w}\right\|_{0} \leq C h^{2}\|\mathbf{w}\|_{2}, \quad \forall \mathbf{w} \in\left(H^{2}(\Omega)\right)^{2}, \\
& \left\|\mathbf{J}_{2 h}^{2} q-q\right\|_{0} \leq C h^{2}\|q\|_{2}, \quad \forall q \in H^{2}(\Omega), \quad\left\|\mathbf{I}_{2 h}^{2} u\right\|_{1} \leq C h^{2}\|u\|_{3}, \quad \forall u \in H^{3}(\Omega), \\
& \left\|\boldsymbol{\Pi}_{2 h}^{2} \mathbf{w}_{h}\right\|_{0} \leq C\left\|\mathbf{w}_{h}\right\|_{0}, \quad \forall \mathbf{w}_{h} \in \mathbf{N}_{h}, \quad\left\|\mathbf{J}_{2 h}^{2} q_{h}\right\|_{0} \leq C\left\|q_{h}\right\|_{0}, \quad \forall q_{h} \in W_{h}, \\
& \left\|\mathbf{I}_{2 h}^{2} v_{h}\right\|_{1} \leq C\left\|v_{h}\right\|_{1}, \quad \forall v_{h} \in V_{0}^{h} .
\end{aligned}
$$

Using these post-processing operators, we can achieve the following global superconvergence for all three dispersive media.

Theorem 4.1. Under the conditions of Theorem 3.2, there holds the following global superconvergent results

$$
\begin{gathered}
\max _{1 \leq n \leq N}\left(\left\|\mathbf{E}^{n}-\mathbf{\Pi}_{2 h}^{2} \mathbf{E}_{h}^{n}\right\|_{0}+\left\|\mathbf{P}^{n}-\mathbf{\Pi}_{2 h}^{2} \mathbf{P}_{h}^{n}\right\|_{0}\right. \\
\left.+\left\|H^{n}-\mathbf{J}_{2 h}^{2} H_{h}^{n}\right\|_{0}+\left\|\nabla\left(u^{n}-\mathbf{I}_{2 h}^{2} u_{h}^{n}\right)\right\|_{0}\right) \\
\leq C\left(h^{2}+\tau\right) .
\end{gathered}
$$


Proof. Notice that

$$
\mathbf{E}^{n}-\Pi_{2 h}^{2} \mathbf{E}_{h}^{n}=\mathbf{E}^{n}-\Pi_{2 h}^{2} \Pi_{h} \mathbf{E}^{n}+\Pi_{2 h}^{2} \Pi_{h} \mathbf{E}^{n}-\Pi_{2 h}^{2} \mathbf{E}_{h}^{n},
$$

using (4.1a)-(4.1e), we have

$$
\left\|\mathbf{E}^{n}-\Pi_{2 h}^{2} \Pi_{h} \mathbf{E}^{n}\right\|_{0}=\left\|\mathbf{E}^{n}-\Pi_{2 h}^{2} \mathbf{E}^{n}\right\|_{0} \leq C h^{2}\left\|\mathbf{E}^{n}\right\|_{2},
$$

and

$$
\left\|\boldsymbol{\Pi}_{2 h}^{2} \boldsymbol{\Pi}_{h} \mathbf{E}^{n}-\boldsymbol{\Pi}_{2 h}^{2} \mathbf{E}_{h}^{n}\right\|_{0}=\left\|\boldsymbol{\Pi}_{2 h}^{2}\left(\boldsymbol{\Pi}_{h} \mathbf{E}^{n}-\mathbf{E}_{h}^{n}\right)\right\|_{0} \leq C\left\|\boldsymbol{\Pi}_{h} \mathbf{E}^{n}-\mathbf{E}_{h}^{n}\right\|_{0} \leq C\left(h^{2}+\tau\right) .
$$

According to (4.2)-(4.4), and using triangle inequality,

$$
\left\|\mathbf{E}^{n}-\boldsymbol{\Pi}_{2 h}^{2} \mathbf{E}_{h}^{n}\right\|_{0} \leq\left\|\mathbf{E}^{n}-\boldsymbol{\Pi}_{2 h}^{2} \boldsymbol{\Pi}_{h} \mathbf{E}^{n}\right\|_{0}+\left\|\boldsymbol{\Pi}_{2 h}^{2} \boldsymbol{\Pi}_{h} \mathbf{E}^{n}-\boldsymbol{\Pi}_{2 h}^{2} \mathbf{E}_{h}^{n}\right\|_{0} \leq C\left(h^{2}+\tau\right) .
$$

Similarly, we have

$$
\begin{aligned}
& \left\|\mathbf{P}^{n}-\mathbf{\Pi}_{2 h}^{2} \mathbf{P}_{h}^{n}\right\|_{0} \leq C\left(h^{2}+\tau\right), \\
& \left\|H^{n}-\mathbf{J}_{2 h}^{2} H_{h}^{n}\right\|_{0} \leq C\left(h^{2}+\tau\right), \\
& \left.\left\|\nabla\left(u^{n}-\mathbf{I}_{2 h}^{2} u_{h}^{n}\right)\right\|_{0}\right) \leq C\left(h^{2}+\tau\right) .
\end{aligned}
$$

The proof can be completed.

\section{Numerical examples}

In this section, we provide a numerical examples to confirm the theoretical analysis. Let the domain $\Omega=[0,1] \times[0,1]$, and the exact solutions

$$
\begin{aligned}
& \mathbf{E}(x, y, t)=\left[-e^{-t} \cos (\pi x) \sin (\pi y), e^{-t} \sin (\pi x) \cos (\pi y)\right], \\
& H(x, y, t)=2 \pi e^{-t}(\cos (\pi x) \cos (\pi y)), \\
& \mathbf{P}(x, y, t)=\left[2 e^{-t} \cos (\pi x) \sin (\pi y),-2 e^{-t} \sin (\pi x) \cos (\pi y)\right], \\
& u(x, y, t)=e^{-t} \sin (\pi x) \sin (\pi y),
\end{aligned}
$$

with the electric conductivity

$$
\sigma(u)=\frac{1}{1+u^{2}}+1
$$

We divide the domain $\Omega$ into $N \times N$ uniform rectangles, and choose $\tau=O\left(h^{2}\right)$. The convergence and superconvergence results of $\mathbf{E}, H, \mathbf{P}$, and $u$ with respect to $t=0.5,1.0$ are listed in Tables 1-8, respectively. It can be seen from Tables 1-4 that $\left\|\mathbf{E}^{n}-\mathbf{E}_{h}^{n}\right\|_{0}$, $\left\|H^{n}-H_{h}^{n}\right\|_{0},\left\|\mathbf{P}^{n}-\mathbf{P}_{h}^{n}\right\|_{0},\left\|u^{n}-u_{h}^{n}\right\|_{1}$ are convergent at optimal rate of $\mathcal{O}(h)$, respectively. From Tables 5-8, we can see that $\left\|\mathbf{E}^{n}-\mathbf{\Pi}_{2 h}^{2} \mathbf{E}_{h}^{n}\right\|_{0},\left\|H^{n}-\mathbf{J}_{2 h}^{2} H_{h}^{n}\right\|_{0},\left\|\mathbf{P}^{n}-\boldsymbol{\Pi}_{2 h}^{2} \mathbf{P}_{h}^{n}\right\|_{0}$, $\left\|u_{h}^{n}-\mathbf{I}_{2 h}^{2} u_{h}^{n}\right\|_{1}$ are of scale $\mathcal{O}\left(h^{2}\right)$, respectively. This data coincide with the theoretical analysis. Moreover, for clarity, we also plot the convergence errors and superconvergent errors using logarithm scales in Figs. 2 and 3. 

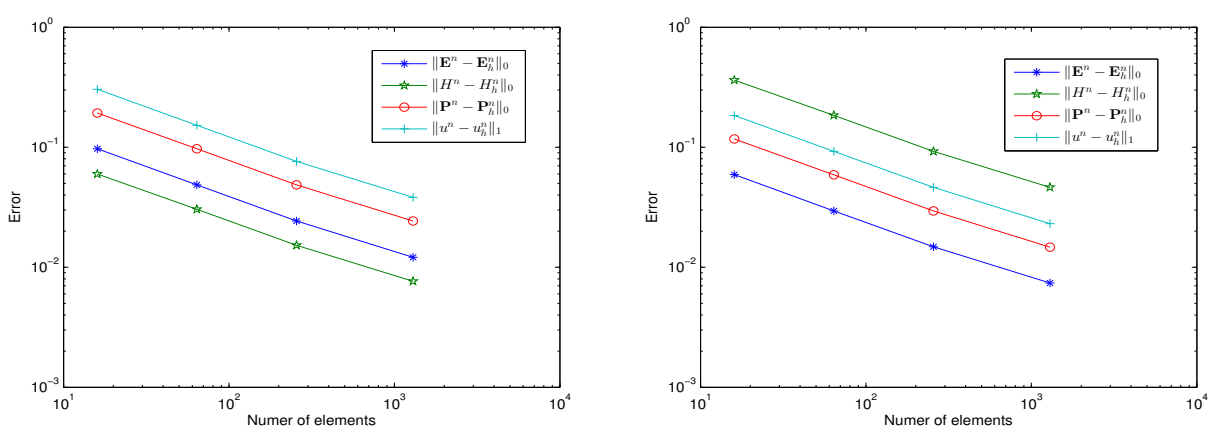

Figure 2: The errors at $t=0.5$ (left) and $t=1.0$ (right).
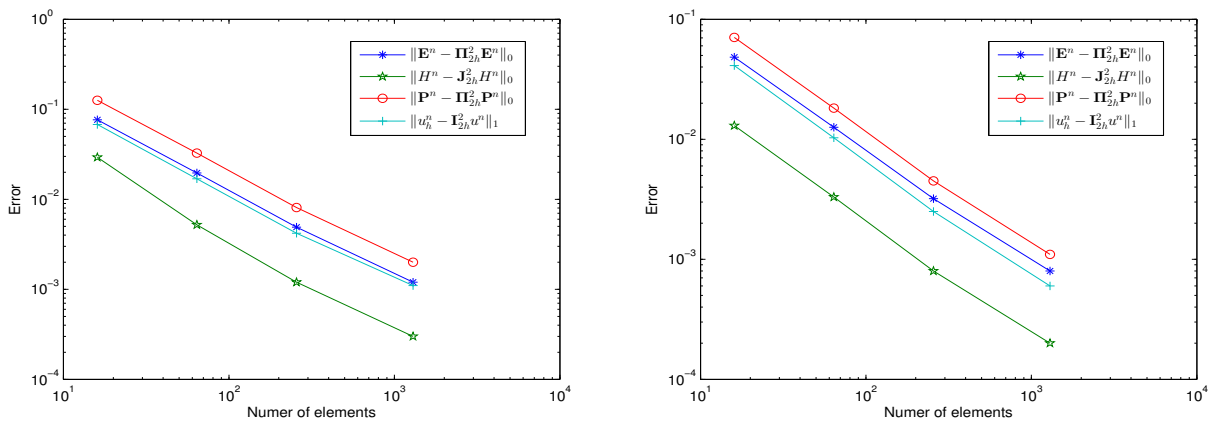

Figure 3: The superconvergent errors at $t=0.5$ (Left) and $t=1$ (right).

Table 1: Convergent results at $t=0.5$.

\begin{tabular}{||ccccccc||}
\hline$N \times N$ & $\left\|\mathbf{E}^{n}-\mathbf{E}_{h}^{n}\right\|_{0}$ & Order & $\left\|H^{n}-H_{h}^{n}\right\|_{0}$ & Order & $\left\|\mathbf{P}^{n}-\mathbf{P}_{h}^{n}\right\|_{0}$ & Order \\
\hline $4 \times 4$ & 0.0971 & - & 0.5982 & - & 0.1929 & - \\
$8 \times 8$ & 0.0486 & 0.9981 & 0.3039 & 0.9771 & 0.0970 & 0.9913 \\
$16 \times 16$ & 0.0243 & 0.9999 & 0.1525 & 0.9943 & 0.0486 & 0.9977 \\
$32 \times 32$ & 0.0121 & 1.0000 & 0.0764 & 0.9986 & 0.0243 & 0.9994 \\
\hline
\end{tabular}

Table 2: Convergent results at $t=0.5$.

\begin{tabular}{||ccccc||}
\hline$N \times N$ & $\left\|u^{n}-u_{h}^{n}\right\|_{0}$ & Order & $\left\|u^{n}-u_{h}^{n}\right\|_{1}$ & Order \\
\hline $4 \times 4$ & 0.0160 & - & 0.3035 & - \\
$8 \times 8$ & 0.0041 & 1.9783 & 0.1525 & 0.9913 \\
$16 \times 16$ & 0.0010 & 1.9950 & 0.0763 & 0.9981 \\
$32 \times 32$ & 0.0003 & 1.9988 & 0.0382 & 0.9995 \\
\hline
\end{tabular}

\section{Acknowledgements}

The first author is supported by NSFC (Nos. 11871441 and 11571389) and the Specialized Research Fund for State Key Laboratory of Space Weather (No. 201916). The second 
Table 3: Convergent results at $t=1.0$.

\begin{tabular}{||ccccccc||}
\hline$N \times N$ & $\left\|\mathbf{E}^{n}-\mathbf{E}_{h}^{n}\right\|_{0}$ & Order & $\left\|H^{n}-H_{h}^{n}\right\|_{0}$ & Order & $\left\|\mathbf{P}^{n}-\mathbf{P}_{h}^{n}\right\|_{0}$ & Order \\
\hline $4 \times 4$ & 0.0592 & - & 0.3627 & - & 0.1172 & - \\
$8 \times 8$ & 0.0295 & 1.0033 & 0.1843 & 0.9769 & 0.0589 & 0.9927 \\
$16 \times 16$ & 0.0148 & 1.0020 & 0.0925 & 0.9942 & 0.0295 & 0.9983 \\
$32 \times 32$ & 0.0074 & 1.0006 & 0.0463 & 0.9985 & 0.0147 & 0.9996 \\
\hline
\end{tabular}

Table 4: Convergent results at $t=1.0$.

\begin{tabular}{||ccccc||}
\hline$N \times N$ & $\left\|u^{n}-u_{h}^{n}\right\|_{0}$ & Order & $\left\|u^{n}-u_{h}^{n}\right\|_{1}$ & Order \\
\hline $4 \times 4$ & 0.0094 & - & 0.1841 & - \\
$8 \times 8$ & 0.0023 & 2.0539 & 0.0924 & 0.9930 \\
$16 \times 16$ & 0.0005 & 2.0067 & 0.0463 & 0.9981 \\
$32 \times 32$ & 0.0001 & 2.0010 & 0.0231 & 0.9995 \\
\hline
\end{tabular}

Table 5: Super-convergent phenomenon at $t=0.5$.

\begin{tabular}{||ccccc||}
\hline$N \times N$ & $\left\|\mathbf{E}^{n}-\mathbf{\Pi}_{2 h}^{2} \mathbf{E}_{h}^{n}\right\|_{0}$ & Order & $\left\|H^{n}-\mathbf{J}_{2 h}^{2} H_{h}^{n}\right\|_{0}$ & Order \\
\hline $4 \times 4$ & 0.0765 & - & 0.0293 & - \\
$8 \times 8$ & 0.0196 & 1.9664 & 0.0052 & 2.2035 \\
$16 \times 16$ & 0.0049 & 1.9903 & 0.0011 & 2.0621 \\
$32 \times 32$ & 0.0012 & 1.9976 & 0.0003 & 2.0164 \\
\hline
\end{tabular}

Table 6: Super-convergent phenomenon at $t=0.5$.

\begin{tabular}{||ccccc||}
\hline$N \times N$ & $\left\|\mathbf{P}^{n}-\boldsymbol{\Pi}_{2 h}^{2} \mathbf{P}_{h}^{n}\right\|_{0}$ & Order & $\left\|u_{h}^{n}-\mathbf{I}_{2 h}^{2} u_{h}^{n}\right\|_{1}$ & Order \\
\hline $4 \times 4$ & 0.1258 & - & 0.0679 & - \\
$8 \times 8$ & 0.0326 & 1.9964 & 0.0169 & 1.9991 \\
$16 \times 16$ & 0.0081 & 2.0004 & 0.0042 & 1.9996 \\
$32 \times 32$ & 0.0020 & 2.0002 & 0.0011 & 1.9999 \\
\hline
\end{tabular}

Table 7: Super-convergent phenomenon at $t=1.0$.

\begin{tabular}{||ccccc||}
\hline$N \times N$ & $\left\|\mathbf{E}^{n}-\mathbf{\Pi}_{2 h}^{2} \mathbf{E}_{h}^{n}\right\|_{0}$ & Order & $\left\|H^{n}-\mathbf{J}_{2 h}^{2} H_{h}^{n}\right\|_{0}$ & Order \\
\hline $4 \times 4$ & 0.0483 & - & 0.0130 & - \\
$8 \times 8$ & 0.0126 & 1.9790 & 0.0033 & 2.2562 \\
$16 \times 16$ & 0.0032 & 1.9943 & 0.0008 & 2.0632 \\
$32 \times 32$ & 0.0008 & 1.9985 & 0.0002 & 2.0172 \\
\hline
\end{tabular}

Table 8: Super-convergent phenomenon at $t=1.0$.

\begin{tabular}{||ccccc||}
\hline$N \times N$ & $\left\|\mathbf{P}^{n}-\boldsymbol{\Pi}_{2 h}^{2} \mathbf{P}_{h}^{n}\right\|_{0}$ & Order & $\left\|u_{h}^{n}-\mathbf{I}_{2 h}^{2} u_{h}^{n}\right\|_{1}$ & Order \\
\hline $4 \times 4$ & 0.0705 & - & 0.0411 & - \\
$8 \times 8$ & 0.0183 & 2.0046 & 0.0102 & 1.9998 \\
$16 \times 16$ & 0.0044 & 2.0012 & 0.0024 & 1.9999 \\
$32 \times 32$ & 0.0011 & 2.0010 & 0.0006 & 2.0000 \\
\hline
\end{tabular}


author is supported by NSFC (No. 11671369). At the same time, the authors gratefully acknowledge the referees for their great efforts and valuable suggestions or questions on our manuscript.

\section{References}

[1] P. Ciarlet AND J. Zou, Fully discrete finite element approaches for time-dependent Maxwell's equations, Numer. Math., 82(2)(1999), pp. 193-219.

[2] Y. CHEN, L. CHEN AND X. ZHANG, Two-Grid method for nonlinear parabolic equations by expanded mixed finite element methods, Numer. Methods Partial Differential Equations, 29(4) (2013), pp. 1238-1256.

[3] P. DebYe, Molecules Polar, Chemical Catalog Co, New York, 1929.

[4] Y. HuANG, J. Li AND C. WU, Superconvergence analysis of second and third order rectangular edge elements with applications to Maxwell's equations, Comput. Methods Appl. Mech. Eng., 329 (2018), pp. 159-176.

[5] Z. TIAN, Y. CHEN AND J. WANG, Superconvergence analysis of bilinear finite element for the nonlinear Schrödinger equation on the rectangular mesh, Adv. Appl. Math. Mech., 10(2) (2018), pp. 468-484.

[6] L. ZHOU, S. SHU AND H. YU, Error estimate and superconvergence of a high-accuracy difference scheme for solving parabolic equations with an integral two-space-variables condition, Adv. Appl. Math. Mech., 10(2) (2018), pp. 362-389.

[7] J. LI, Y. HuANG AND W. YANG, An adaptive edge finite element method for electromagnetic cloaking simulation, J. Comput. Phys., 249 (2013), pp. 216-232.

[8] Y. HuANG, J. LI AND W. YANG, Superconvergence of mixed finite element approximations to 3-D Maxwells equations in metamaterials, J. Comput. Phys., 230(22) (2011), pp. 8275-8289.

[9] J. Li AND Y. HuANG, Time-Domain Finite Element Methods for Maxwell's Equations in Metamaterials, Springer Science and Business Media, 2012.

[10] J. LI AND Z. FANG, Analysis and application of stochastic collocation methods for Maxwell's equations with random inputs, Adv. Appl. Math. Mech., 10(6) (2018), pp. 1305-1326.

[11] J. WANG, Z. XIE AND C. CHEN, Implicit DG method for time domain Maxwell's equations involving metamaterials, Adv. Appl. Math. Mech., 7(6) (2015), pp. 796-817.

[12] Y. HUANG, J. LI AND Y. LiN, Finite element analysis of Maxwell's equations in dispersive lossy bi-isotropic media, Adv. Appl. Math. Mech., 5(4) (2013), pp. 494-509.

[13] C. LiU, S. SHU, Y. HuANG, L. ZHONG AND J. WANG, An iterative two-grid method of a finite element PML approximation for the two dimensional Maxwell problem, Adv. Appl. Math. Mech., 4(2) (2012), pp. 175-189.

[14] Q. LiN AND J. LIN, Finite Element Methods: Accuracy and Improvement, Beijing Science Press, Beijing, 2006.

[15] Q. LiN AND N. YAN, Global superconvergence for Maxwell's equations, Math. Comput., 69 (1999), pp. 159-176.

[16] Q. LIN AND N. YAN, Superconvergence of mixed finite element methods for Maxwell's equations, Gongcheng Shuxue Xuebao, 13(12) (1996), pp. 1-10.

[17] Q. LIN AND J. LIN, Global superconvergence of the mixed finite element methods for 2-D Maxwell's equations, J. Comput. Math., 21(5) (2003), pp. 637-646.

[18] Q. LIN, L. TOBISKA AND A. ZHOU, Superconvergence and extrapolation of non-conforming low order finite elements applied to the Poisson equation, IMA J. Numer. Anal., 25(1) (2005), pp. 160- 
181.

[19] A. LOMBARDI, Interpolation error estimates for edge elements on anisotropic meshes, IMA J. Numer. Anal., 31(4) (2011), pp. 1683-1712.

[20] H. LIU AND N. YAN, Superconvergence analysis of the nonconforming quadrilateral linearconstant scheme for Stokes equations, Adv. Comput. Math., 29 (2008), pp. 375-392.

[21] H. GAO, Optimal error analysis of Galerkin FEMs for nonlinear Joule-heating equations, J. Sci. Comput., 58(3) (2014), pp. 627-647.

[22] K. KunZ And R. Luebbers, The Finite Difference Time-Domain Method for Electromagnetics, CRC Press, Boca Raton, FL, 1993.

[23] P. MonK, Finite Element Methods for Maxwell's Equations, Oxford University Press, New York, 2003.

[24] P. MONK, Superconvergence of finite element approximations to Maxwell's equations, Numer. Methods for Partial Differential Equations, 10(6) (2010), pp. 793-812.

[25] A. PANI, An $\mathrm{H}^{1}$-Galerkin mixed finite element method for parabolic partial differential equations, SIAM J. Numer. Anal., 35(2) (1998), pp. 721-727.

[26] Z. QIAO, C. YAO AND S. JIA, Superconvergence and extrapolation analysis of a nonconforming mixed finite element approximation for time-harmonic Maxwell's equations, J. Sci. Comput., 46(1) (2011), pp. 1-19.

[27] D. SHI AND C. YAO, Nonconforming finite element approximation of time-dependent Maxwell's equations in Debye medium, Numer. Methods Partial Differential Equations, 30(5) (2014), pp. 1654-1673.

[28] D. SHI AND H. YANG, Superconvergent estimates of conforming finite element method for nonlinear time-dependent Joule-heating equations, Numer. Methods Partial Differential Equations, 34 (2018), pp. 336-356.

[29] D. SHI, F. WANG AND Y. ZHAO, Superconvergence analysis and extrapolation of quasi-Wilson nonconforming finite element method for nonlinear Sobolev equations, Acta. Math. Appl. Sin., 29(2) (2013), pp. 403-414.

[30] D. SHI, F. YAN AND J. WANG, Unconditional superconvergence analysis of a new mixed finite element method for nonlinear Sobolev equation, Appl. Math. Comput., 274 (2016), pp. 182-194.

[31] C. YAO, J. LI AND D. SHI, Superconvergence analysis of nonconforming mixed finite element methods for time-dependent Maxwell's equations in isotropic cold plasma media, Appl. Math. Comput., 219(2) (2013), pp. 6466-6472.

[32] C. YAO AND S. JIA, Asymptotic expansion analysis of nonconforming mixed finite element methods for time-dependent Maxwell's equations in Debye medium, Appl. Math. Comput., 229(229) (2014), pp. 34-40.

[33] C. YAO, The solvability of coupling the thermal effect and magnetohydrodynamics field with turbulent convection zone and the flow field, J. Math. Anal. Appl., 476(2) (2019), pp. 495-521.

[34] Q. HuANG, S. JIA, F. XU, Z. XU AND C. YAO, Solvability of wave propagation with Debye polarization in nonlinear dielectric materials and its finite element methods approximation, Appl. Numer. Math., 146 (2019), pp. 145-164.

[35] H. YIN, Existence and regularity of a weak solution to Maxwell's equations with a thermal effect, Math. Methods Appl. Sci., 29(10) (2010), pp. 1199-1213.

[36] Y. ZHANG AND D. SHI, Superconvergence of an $\mathrm{H}^{1}$-Galerkin nonconforming mixed finite element method for a parabolic equation, Comput. Math. Appl., 66(11) (2013), pp. 2362-2375. 